\title{
Understanding the chapati making attributes of the Indian wheats - I: The physico-chemical basis
}

\author{
Satish Kumar* \\ Virinder Singh Sohu \\ Punjab Agricultural University, Ludhiana - 141004 (Punjab), India \\ Satish Kumar Gupta \\ Punjab Agricultural University, Ludhiana - 141004 (Punjab), India \\ Rajinder Pal Singh \\ Punjab Agricultural University, Ludhiana - 141004 (Punjab), India \\ Navtej Singh Bains \\ Punjab Agricultural University, Ludhiana - 141004 (Punjab), India \\ *Corresponding author. E-mail: kumarsatish227@gmail.com
}

${ }^{1}$ ICAR - Indian Institute of Wheat and Barley Research, Karnal - 132001(Haryana), India

\begin{abstract}
The research project was largely built around the tall traditional cultivars of the predwarfing era, which were known to excel for chapati quality. These included C 306, C 518 and $\mathrm{C} 273$. The few cultivars that had emanated from crosses of these superior chapati quality wheats with dwarf wheats formed another important component of this set and included WG 357, PBW 175, PBW 154, PBW 226, Lok 1 among others. Asecond set of materials, three backcross recombinant populations $\left(B C_{1} F_{5}\right.$ generation) derived from $C$ 273/PBW 343//PBW 343 (70 lines), C 306/PBW 534//PBW 534 (70 lines) and C 518/ PBW 343//PBW 343 (80 lines) were also studied to arrive at some conclusion. Various physico-chemical characters(Grain appearance score,Grain hardness,Test weight,1000 grain weight,Yellow berry,Moisture content,Protein content,Gluten content, Gluten index, Sedimentation value, Phenol Test, Carotenoids, Sugar content, Diastatic activity, Falling Number) and chapati-makingscores were evaluated. Grain hardness seems to have a clear role in chapati quality with a correlation coefficient of $0.34,0.35$ and 0.17 observed in different recombinant populations.More consistent correlation was found for grain appearance ranging from 0.26 to 0.36 in the populations.Consistent high positive correlations have showed up for diastase activity, which ranged from 0.32 to 0.46 . This consistent behaviour is a strong evidence for the role of this trait in chapati making quality.Diastase activity emerges as a more consistent and stronger contributor to chapati making quality. Phenol score may not serve as a suitable indicator of chapati quality.
\end{abstract}

Keywords: Chapati making quality, Diastase activity, Phenol score, Total and reducing sugars, Wheat

\section{INTRODUCTION}

In spite of the predominant position of chapati in the Indian diet, relatively small numbers of studies have been carried out to investigate and improve the quality of the chapaties. The present study was aimed at understanding the basis of chapati quality in wheat by study of two sets of genotypes. One was assembled from cultivars and stocks known for end product quality or specific quality indices, and the other was based on recombinant inbred lines derived from specific crosses having a parent excelling in chapati quality. The study is divided in to three components for the ease of presenting the results. In the first part we describe the results obtained from the physic-chemical observations on a set of genotypes and inferences

\section{Article Info}

DOI:10.31018/jans.v10i2.1739

Received: January 18, 2018

Revised: February 15, 2018

Accepted: April 4, 2018

\section{How to Cite}

Kumar, S. et al. (2018).

Understanding the chapati making attributes of the Indian wheats - I: The physico-chemical basis. Journal of Applied and Natural Science, 10(2): $572-592$ 
(Austin and Ram 1971). While studying the genetic basis of protein content, Gupta et al (1999) studied 100 RILs (recombinant inbred lines) from a cross between $\mathrm{PH} 132$ having high protein content (13.5 per cent) and WL 711 having lower protein content (9.7 per cent). Out of 232 STMS primer pairs used in the study, 57 were found to be polymorphic for the parents. Irvine and Bains (1965) studied the improved wheat varieties of Punjab and reported that our wheat produced short dough which was not suitable for bread making. Various physico-chemical characteristics other than grain hardness and protein content, such as grain appearance, sedimentation value, gluten content, diastatic activity, sugar content, damaged starch content and phenol reaction etc are known to have either direct or indirect effect on the chapati quality (Naik et al., 2010).

Upretty and Abrol (1972) reported a significant variation in the total sugar content in the whole meals of different varieties. The authors reported changes in the reducing and non reducing sugar contents during the chapati making process. They also advocated that determination of diastatic power can serve as a useful index of sugars released during the processing stages. Finney et al (1973) compared the Indian and newly introduced Mexican wheats and reported that in Indian wheats the test weight averaged $79 \mathrm{~kg} /$ hectolitre and 1000 grain weight ranged from 28.0 to $52.3 \mathrm{~g}$. They reported that the flour protein ranged from 8.0-13.3 per cent with an average of 10.7 per cent. The average sedimentation value for Indian wheats was observed to vary from 20.5 to $36.5 \mathrm{cc}$ with an average of $27.5 \mathrm{cc}$. Singh et al (1978) involved five different varieties of bread wheat for their study which included WG 357, a variety known to produce very good chapaties. On the whole the proteolytic activity in Indian wheat was reported to be low. The effect of flour milling conditions on the quality of Indian unleavened flat bread and effect of wheat meal granulation, damaged starch and protein contents, on the quality of chapati was studied by Sidhu et al (1988). It was shown that higher water absorption led to enhanced moisture retention in chapaties and viceversa. The authors suggested that finer flours having more than 70 percent water absorption were more suitable for producing chapaties of desirable texture. Again in 1990 the authors studied the starch gelatinization in relation to the preparation of Indian unleavened flat breads. Rao et al (1989) studied the effect of damaged starch on the functional quality characteristics of whole meal flour used for chapati-making. Damaged starch in the flour was positively correlated to the diastatic activity and flour water absorption. The study confirmed that the rheological characteristics of whole wheat flour were also influenced by the damaged starch as indicated by its significant correlation to dough development time, extensibility, resistance to extension, cohesiveness and adhesiveness. The content of gluten in the bread wheat varieties ranged from 6.4 per cent to 9.3 per cent. Mishra (1998) found that the negligible activity of Tyrosinage activity in C 306, NP 4, Raj 3077 and other good chapati quality wheats showed a positive relation with phenol test. Syed et al (1991) also gave a brief review of physical parameters, gluten content, protein and mineral contents of Indian wheat. Saxena et al (1997) tried to correlate the physico-chemical and rheological characteristics of wheat flour with the tandoori roti quality, using eight commercial wheat cultivars (PBW 154, PBW 175, PBW 229, PBW 138, WL 1562, CPAN 3004, GW 180 and K 8804) grown at one location. Varieties with high water absorption capacity gave roties with better quality. Medium hard wheats with a Glu-1 score of 6 were best suited for the preparation of tandoori roti. Similar studies of quality parameters of Indian wheat varieties (Sekhon et al 1976, Singh et al 1983, Syed et al 1990, Supekar et al 2005, Gill et al 2006) have indicated that there is a wide variation for various physico-chemical characteristics but majority of the varieties produce average to good chapaties. Ahmed et al. (2015) studied physicochemical and rheological properties of soft wheat flours obtained from different wheat varieties grown in Pakistan, Ukraine and India. The rheological behaviour of Indian wheat flour showed high water absorption, high dough stability and less degree of softening.

Most of the studies in literature focused on the released cultivars or advanced lines developed for end use quality, however the present study was built around a set of cultivars/genotypes and three recombinant inbred populations. The results are indicative of the physic-chemical basis of chapatimaking quality of wheat.

\section{MATERIAL AND METHODS}

Selection of plant material - set I: The research project was largely built around the tall traditional cultivars of the pre-dwarfing era, which were known to excel for chapati quality. These included C 306, C 518 and C 273. The few cultivars that had emanated from crosses of these superior chapati quality wheats with dwarf wheats formed another important component of this set and included WG 357, PBW 175, PBW 154, PBW 226, Lok 1 and others as mentioned in the Table 1. All the plant material was sown in a randomized complete block design in three replications with a plot size of $2 \mathrm{~m}$ length and four rows per plot and was replicated three times.

Selection of plant material - set II: To represent the second set of materials, three backcross recombinant populations $\left(\mathrm{BC}_{1} \mathrm{~F}_{5}\right.$ generation) derived from C 273/PBW 343//PBW 343 (70 lines), C 306/ PBW 534//PBW 534 (70 lines) and C 518/PBW 
343//PBW 343 (80 lines) were studied. The four parental lines were included in the analysis and these also formed a part of first set, allowing their placement in the overall range of variation for various traits. Approximately $100 \mathrm{BC}_{1} \mathrm{~F}_{2}$ and $\mathrm{BC}_{1} \mathrm{~F}_{3}$ plant to row progenies (derived from independent random $\mathrm{BC}_{1} \mathrm{~F}_{1}$ plants) harvested at Keylong during off season were used to generate $\mathrm{BC}_{1} \mathrm{~F}_{3}$ and $\mathrm{BC}_{1} \mathrm{~F}_{4}$ populations sown at Ludhiana during main crop season. Six ears were randomly taken from each progeny and a plot of six rows of $1 \mathrm{~m}$ each per progeny was sown. Five to ten single plants were harvested from each progeny. Seed harvested from single plant of each progeny was multiplied at Keylong during the off season. Seed of progenies multiplied at Keylong were used for growing $\mathrm{BC}_{1} \mathrm{~F}_{4}$ and $\mathrm{BC}_{1} \mathrm{~F}_{5}$ generations during main crop season. The progenies were sown in a randomized complete block design with a plot size of $2 \mathrm{~m}$ length and 2 rows, with three replications. Parental checks were also used in the trail. The bulk-harvested progenies were evaluated for various physico-chemical characters and chapatimaking.

Observations recorded: Data recording in field and in the laboratory was carried out on the following characteristics:

Grain appearance score: It was evaluated subjectively out of a maximum score of 10 , giving due weightage to the grain size, shape, colour and lustre.

Grain hardness: The grain hardness was measured by using the grain hardness tester supplied by M/S Ogawa Seiki Co. Ltd., Japan by crushing randomly taken ten grains one by one. The mean force $(\mathrm{kg})$ required to crush the grain was recorded.

Test weight: This was determined using the apparatus developed by the Directorate of Wheat Research (DWR), Karnal, which employs a standard container of $100 \mathrm{ml}$ capacity (Mishra et al 1998). The grains were weighed and the test weight expressed in $\mathrm{kg} / \mathrm{hl}$.

1000-grain weight: 250 kernels were counted in duplicate from a random lot of each variety and weighed. The average weight obtained was multiplied by four and expressed in grams.

Yellow berry: Recorded as percentage by weight after manually separating the mottled grains from 1000-grain sample.

Moisture content: The moisture content was estimated using the whole grain analyzer Infratec 1241 supplied by M/S Foss Analytical AB, Sweden. The instrument uses the near infrared light transmitted through the grains. The grain samples are scanned in the range of 850 to $1050 \mathrm{~nm}$ with a bandwidth of $7 \mathrm{~nm}$ and there are 100 data points per scan. The results are displayed as percent.

Protein content: The grain protein content was estimated using the whole grain analyzer Infratec
1241 supplied by M/S Foss Analytical AB, Sweden. The instrument uses the near infrared light transmitted through the grains. The grain samples are scanned in the range of 850 to $1050 \mathrm{~nm}$ with a bandwidth of $7 \mathrm{~nm}$ and there are 100 data points per scan. The results are displayed as percent protein content.

Gluten content and Gluten index: The gluten content and gluten index were evaluated using Glutomatic 2100 system supplied by M/S Perten, Germany. The instrument employs a $10 \mathrm{~g}$ sample of whole meal using the AACC method to determine wet gluten content. The wet gluten was then centrifuged to get strong and weak gluten fractions, which were used to calculate the gluten index. Total gluten was then dried in the Glutork gluten drier and the weight expressed as percent. The gluten index was expressed as the percent wet gluten retained inside the centrifuge cassette.

Sedimentation value: The SDS sedimentation values of samples were determined by employing the method given by Axford et al (1979). A sample weight of $6 \mathrm{~g}$ and a rest period of $20 \mathrm{~min}$ were employed.

Phenol test: The phenol reaction of the wheat genotypes was determined by soaking 15-20 grains of each sample in distilled water for 15-16 hours in Petri plates. After that the water was drained off and 1 per cent solution of phenol was added to the grains so that only three fourth of the grain is covered by the solution. The Petri plates were covered and kept for 4 hour. After 4 hours the phenol solution was also drained off and the grains were dried of filter paper for 30 minutes. A subjective score (out of 10 ) was given to each genotype based on the colour after drying. Higher score was given to the grains with darker intensity of the colour.

Carotenoids: Standard AACC calorimetric method was used to determine the amount of carotenoids in the wheat wholemeal for all the genotypes. $4 \mathrm{~g}$ wholemeal was taken in a $125 \mathrm{ml}$ reagent bottle and $20 \mathrm{ml}$ of water saturated n-butanol was added to it. The contents were mixed properly and kept in dark for 16 hours. The contents were then filtered and the extract transferred to standard test tubes. Light transmission of the extract was measured at $440 \mathrm{~nm}$ using spectrophotometer and recorded as optical density (O.D.).

The amount of carotenoids was calculated using the following formula:

Carotenoids $(\mathrm{ppm})=[($ O.D. $X$ 23.5366) + 0.0105]

Sugar content: AACC (1990) approved method was followed to determine the reducing and nonreducing sugar contents. The sugars were extracted from $5.7 \mathrm{~g}$ of flour in sodium acetate buffer. The proteinacious material was precipitated by the addition of sodium tungstate $(12.0 \%)$. The contents were mixed and filtered. From the filtrate, $5 \mathrm{ml}$ ali- 
quot was taken and the reducing sugars were determined by potassium ferricyanide method. The total sugars were also estimated by potassium ferricyanide method but the $5 \mathrm{ml}$ aliquot was first hydrolyzed by immersing in boiling water bath for $15 \mathrm{~min}$. The difference between the total and reducing sugars gave the content of non-reducing sugars. Reducing sugars were expressed as per cent maltose whereas non-reducing as per cent sucrose.

Diastatic activity: It was determined using AACC (1990) approved method employing $5 \mathrm{~g}$ of flour sample. The sample was incubated with $46 \mathrm{ml}$ of acetic acid sodium acetate buffer $(\mathrm{pH} \mathrm{4.6-4.8)} \mathrm{for}$ $1 \mathrm{hr}$ at $30^{\circ} \mathrm{C}$. The enzyme action was terminated by adding $2 \mathrm{ml}$ of sulphuric acid (10\%) followed by the addition of $2 \mathrm{ml}$ of $12 \%$ sodium tungstate solution. The contents were filtered through Whatman No.4 filter paper and a $5 \mathrm{ml}$ aliquot was taken for maltose determination by the potassium ferricyanide method. The results were expressed as percent maltose produced.

Falling number value (sec): The Falling number values were estimated using the Starch Master supplied by New Port Scientific, Australia using the following procedure: Allow the instrument to warm up for $30 \mathrm{~min}$, weigh $4.0 \mathrm{~g}$ (14\% moisture basis) of whole meal in a consister and add 25.0 $\mathrm{ml}$ of distilled water into the consister. Place the paddle into the consister and vigorously jog the blade through the sample up and down 10 times or until it mixes uniformly. Insert the consister into the pre-adjusted instrument using the 'FNE' profile given below:

$\begin{array}{lll}\begin{array}{l}\text { Time } \\ \text { 00:00:00 }\end{array} & \text { Type } & \text { Value } \\ 95^{\circ} \mathrm{C}\end{array}$

The measurement cycle was initiated by depressing the motor tower of the instrument. The consister was removed on completion of test and discarded. Falling/stiring number values displayed at the end of the test were recorded.

XV) Chapati-making characteristics: For baking chapaties the method used in the quality laboratory Department of Plant Breeding and Genetics was employed (Kumar et al, 2018). The chapati score was calculated using the parameters Dough stickiness (5), Puffing of chapatti (5), Texture of chapati (5), Color of chapati (5), Taste of chapati (5), Flavor of chapati (5)and Texture of chapati after $2 \mathrm{hrs}(5)$. The total score was finally calculated out of a maximum of ten.

\section{Data analysis}

Analysis of variance: The material had been planted in a randomized complete block design. The analysis of variance for different traits was done as per the following model:

Equation $\mathrm{I}: \mathrm{Y}_{\mathrm{ij}}=\mathrm{m}+\mathrm{t}_{\mathrm{i}}+\mathrm{b}_{\mathrm{j}}+\mathrm{e}_{\mathrm{ij}}$

$Y_{i j}=$ observation obtained from the $\mathrm{i}$-th treatment and j-th block.

$\mathrm{m}=$ general mean

$t_{i}=$ the effect of $i$-th treatment

$b_{j}=$ the effect of $j$-th block

$\mathrm{e}_{\mathrm{ij}}=$ error associated with $\mathrm{i}$-th treatment and j-th block

The analysis of variance based on the above model takes the following form:

Where, $r=$ no. of replications

\begin{tabular}{lllll}
\hline Source & d. f. & $\begin{array}{l}\text { Sum of } \\
\text { Squares }\end{array}$ & $\begin{array}{l}\text { Mean } \\
\text { sum of } \\
\text { squares }\end{array}$ & $\begin{array}{l}\text { F- } \\
\text { ratio }\end{array}$ \\
\hline $\begin{array}{l}\text { Repli- } \\
\text { cations }\end{array}$ & $r-1$ & RSS & MSR & $\begin{array}{l}\text { MSR/ } \\
\begin{array}{l}\text { Treat- } \\
\text { ments }\end{array}\end{array}$ \\
Error & $(r-1)(g-1)$ & SSE & MSE & MSE \\
\hline
\end{tabular}

puted as follows:

$\mathrm{g}=$ no. of genotypes

CD to compare two genotypes was com-

$$
C D=\sqrt{\frac{2 M S E}{r}} \times t_{a}(r-1)(g-1)
$$

Where, $a=$ level of significance

Correlation coefficients: The correlation coefficient ( $r$ ) between two different observations (say $X$ $\& Y$ ) was calculated using the following formula: Equation II:

$$
r_{x y}=\frac{\sum\left(x_{i}-\bar{x}\right)\left(y_{i}-\bar{y}\right)}{(n-1) s_{x} s_{y}}
$$

Where: $x$ and $y=$ means of variables $\mathrm{s}_{\mathrm{x}}$ and $\mathrm{s}_{\mathrm{y}}=$ standard deviations of $\mathrm{x}$ and $\mathrm{y}$ $\mathrm{n} \quad=$ population size.

\section{RESULTS AND DISCUSSION}

Analysis of genetic variation for chapati quality and its correlation with various physic-chemical characteristics was the major objective of the present study. It would be instructive to first discuss the results from the set of cultivars and genetic stocks and then their confirmation based on the data of backcross derived recombinant populations.

Results from the set of cultivars and genetic stocks: Each genotype was subjected to chapati making tests using the standard method.Chapati score is a composite trait and is based on dough handling (stickiness of the dough), puffing of chapati, texture, taste, flavour and colour of the chapati. Conventionally, a chapati score above 8 (out of 10$)$ represents excellent chapati quality.

Significant genotypic differences were observed 
Table 1. List of varieties and genetic stocks used in the study.

\begin{tabular}{|c|c|c|}
\hline S.N. & Basis & Genotypes \\
\hline 1 & $\begin{array}{l}\text { Tall wheats known for } \\
\text { chapati quality }\end{array}$ & $\begin{array}{l}8 A \\
9 D \\
\text { C } 306 \\
\text { C } 273 \\
\text { C } 591 \\
\text { C } 518 \\
\text { WG } 357\end{array}$ \\
\hline 2 & $\begin{array}{l}\text { High yielding present day } \\
\text { wheats }\end{array}$ & $\begin{array}{l}\text { PBW } 343 \\
\text { PBW } 502 \\
\text { PBW } 509 \\
\text { PBW } 533 \\
\text { PBW } 550 \\
\text { DBW } 16\end{array}$ \\
\hline 3 & $\begin{array}{l}\text { Good chapati quality } \\
\text { wheat varieties (released) }\end{array}$ & $\begin{array}{l}\text { PBW } 154 \\
\text { PBW } 175 \\
\text { PBW } 226 \\
\text { Lok } 1 \\
\text { HD } 2793\end{array}$ \\
\hline 4 & $\begin{array}{l}\text { High yielding lines with } \\
\text { good grains }\end{array}$ & $\begin{array}{l}\text { PBW } 534 \\
\text { PBW } 531 \\
\text { PBW } 554 \\
\text { HI } 1418 \\
\text { HI } 1479\end{array}$ \\
\hline 5 & Genetic stocks & $\begin{array}{l}\text { Pusa } 5-3 \text { (High protein and Lysine) } \\
\text { DI } 105 \text { (C } 591+\text { rht } 3) \\
\text { DI } 9 \text { (C } 306+\text { rht } 1) \\
\text { WH } 423 \text { (High protein) } \\
\text { WH } 595 \text { (High protein) } \\
\text { WH } 712 \text { (High sedimentation value) } \\
\text { K } 0123 \text { (High protein) } \\
\text { KYZ K2K-13 (High protein) } \\
\text { WH } 800 \text { (High protein and sedimentation value) } \\
\text { WH } 1003 \text { (High sedimentation value) }\end{array}$ \\
\hline 6 & $\begin{array}{l}\text { High protein winter wheat } \\
\text { stock }\end{array}$ & Glupro \\
\hline
\end{tabular}

for chapati score of the genotypes in both the seasons (Table $2 \mathrm{a}$ and $\mathrm{b}$ ). Keeping aside the very low chapati score of unadapted genotype Glupro (5.3), the mean chapati score (Table 3 ) ranged from 7.2 in 'Pusa 5-3' to 8.1 in case of ' $C$ 306' during first year. The chapati score showed variation between the groups of genotypes, with the tall wheats of pre dwarfing era excelling over other groups. The tall varieties not only established themselves as a distinct group with highest chapati score but the next numerical best score (7.8 for both DI 9and DI 105) also went to the derivatives of C 306 and C 591. The commercial high yielding cultivars gave intermediate chapati scores, significantly inferior to the best tall wheat i.e., C 306. Similar was the case of genetic stocks and other varieties which showed chapati scores well below the best entry i.e., C 306. Stocks excelling in one or more quality component (e.g. K 0123, WH 595, Pusa 5-3 for high protein content and $\mathrm{WH} 712, \mathrm{WH} 800$ for high sedimentation value) did not excel for chapati quality. The advanced lines (PBW 531, PBW 534, PBW 554) used in the set performed almost similar to the commercially released varieties. On the whole the genetic stocks DI 9, DI 105 and WH 423 with chapati scores of $7.8,7.8$ and 7.7 respectively were adjudged to be at par with the best cultivar $\mathrm{C}$ 306.In second year, the mean chapati score (Table 4) ranged from 7.1 in case of 'PBW 502' to 8.1 in case of ' $C$ 306' and ' $C$ 518'. The tall varieties were again found significantly superior to all other groups, thus confirming their status as best chapati wheats. The commercial wheat group gave intermediate score with PBW 343 (7.6) having highest score in the group. DBW 16 (having a perfect ' $G / u$ ' score of 10) and PBW 533 were included in the set as they are identified for their excellent bread making properties. These varieties did not fare well for chapati making giving a score of 7.4 only. Among the released varieties known for chapati quality, Lok 1 (chapati score 7.9) performed better than others in the group though slightly lower than C 306 . Thus Lok 1 was able to express good chapati quality outside Central Zone also, where it is recommended for cultivation. Similarly $\mathrm{HI} 1418$ also registered a chapati quality score of 7.8, at par with C 306. PBW 154 (7.2), 
Satish Kumar et al. / J. Appl. \& Nat. Sci. 10 (2): 572 - 592 (2018)

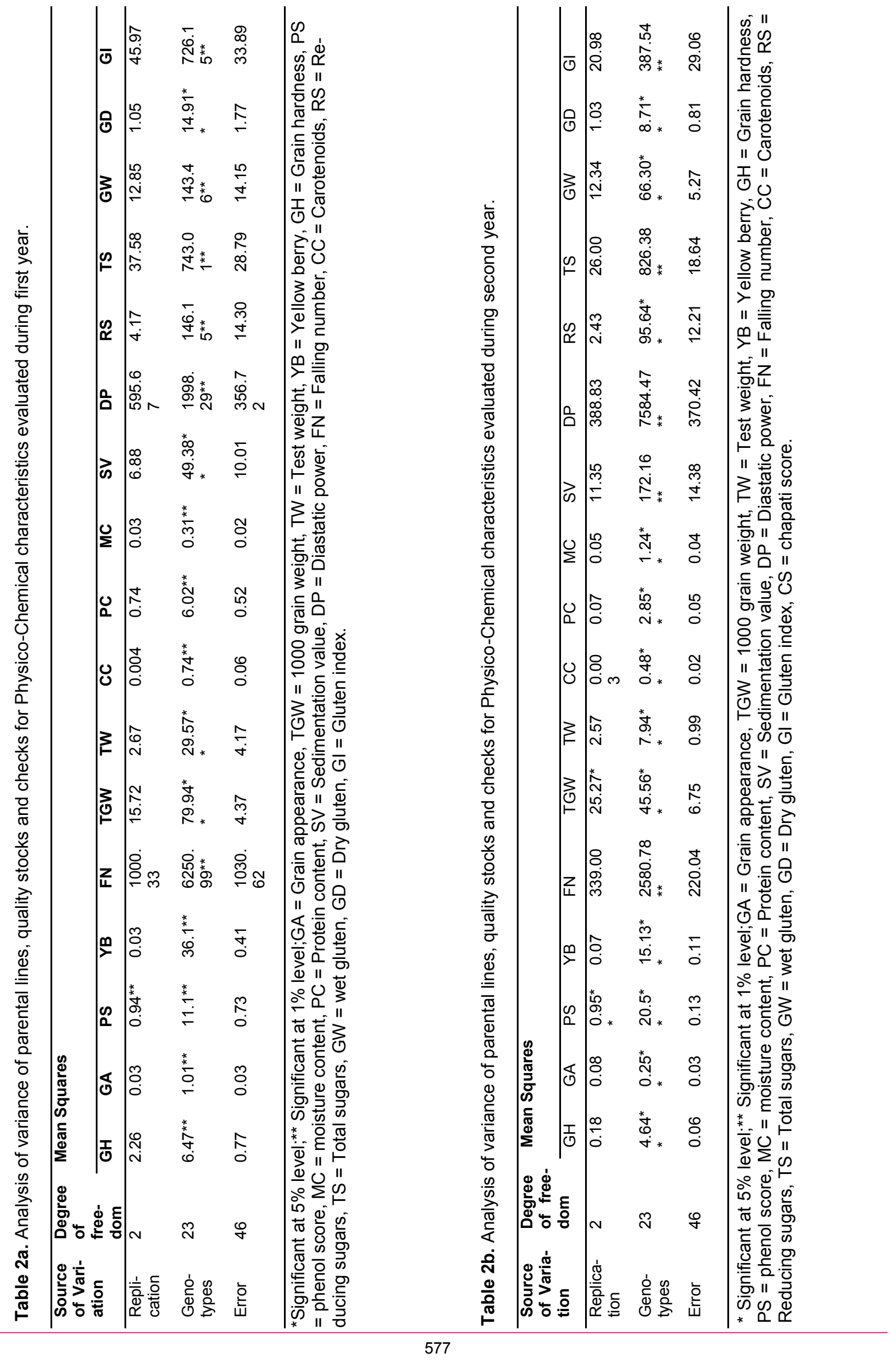




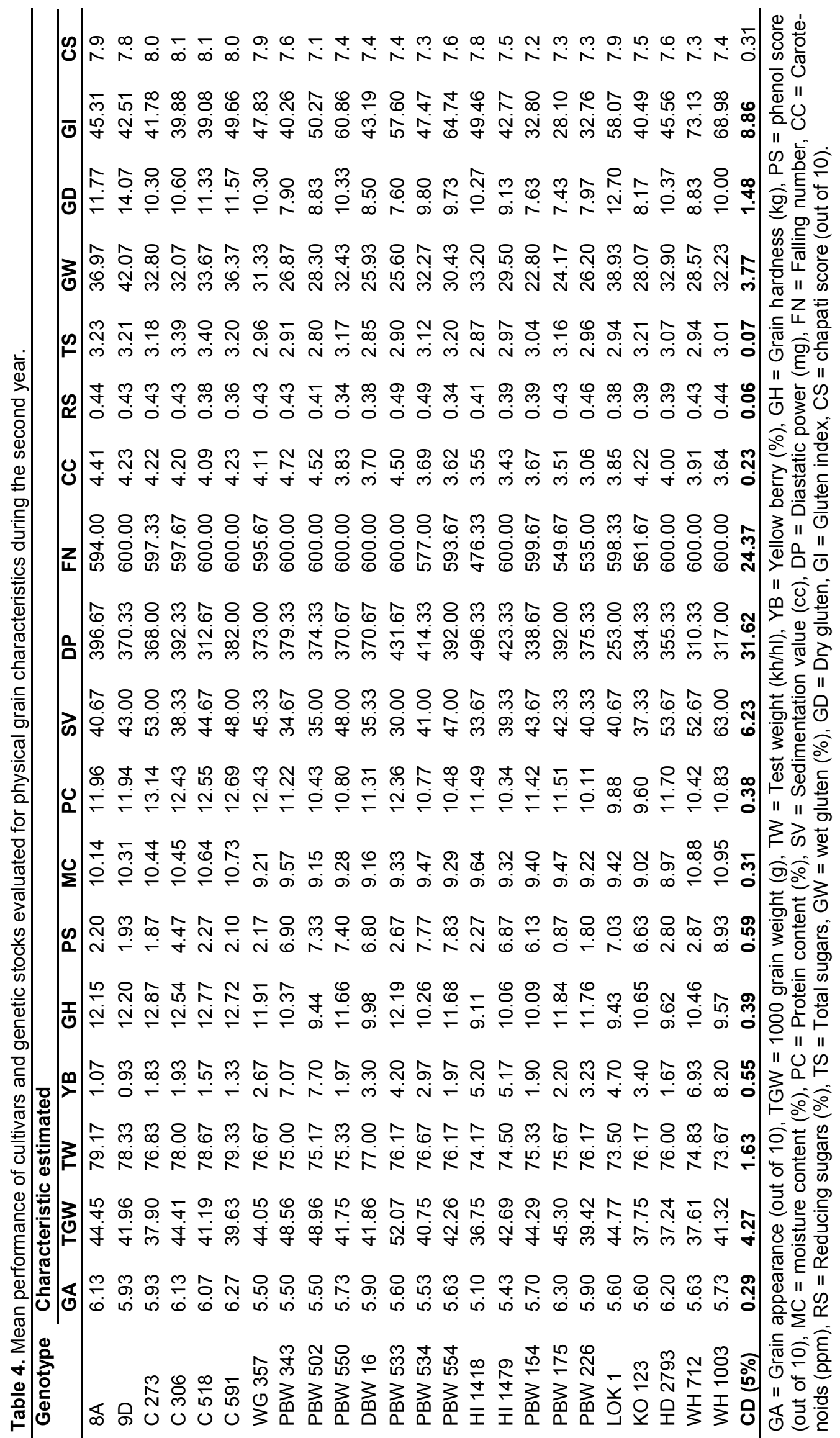




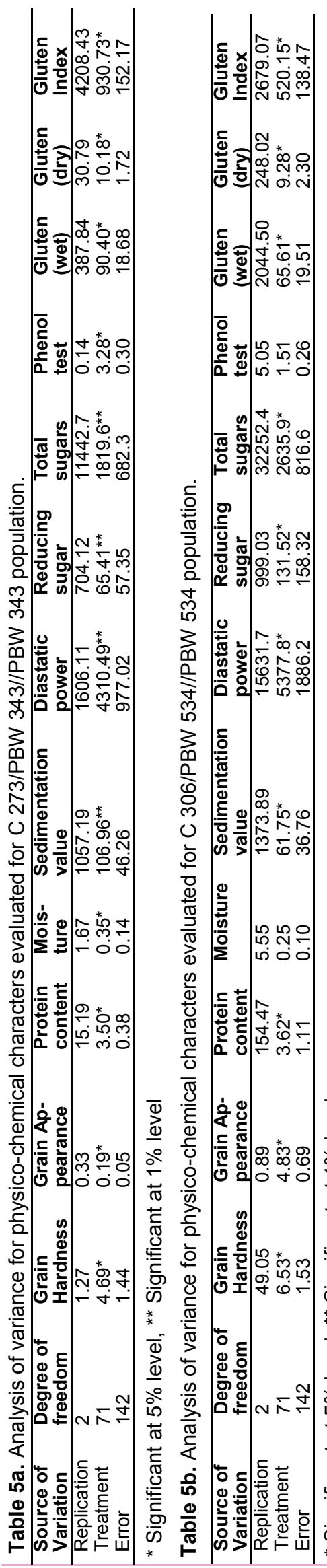

Satish Kumar et al. / J. Appl. \& Nat. Sci. 10 (2): 572 - 592 (2018) group with respect to chapati quality. These genotypes are regarded to be superior for chapati quality among released semi-dwarf varieties and PBW 175 has C-306 as a parent. The genetic stocks, on the other hand again failed to give good chapaties thus emphasizing the fact that good chapati quality resulted from a combination of different component traits and not because of one or two traits. The advanced breeding lines in this season again showed an intermediate chapati score.

Significant genotypic differences were observed for all the physico-chemical characteristics evaluated during the course of present study (Table 2). The mean performance of genotypes is given in table 3 and table 4 . The grain appearance score was best in tall wheat varieties owing to their lustrous grains and attractive colour. Glupro was given a score of 3.57 owing to its highly shriveled and unattractive grains. A minimum score of 5.23 for was given to ' $\mathrm{K} 0123$ ' and a maximum of 6.6 to ' $\mathrm{C}$ 591 ' in first year. In second year ' $\mathrm{HI}$ 1418' was given a minimum score of 5.1 where as 'PBW 175' was given maximum score of 6.3. The values of test weight among the genotypes ranged from $75.00 \mathrm{~kg} / \mathrm{hl}$ for 'WH 423' to $83.00 \mathrm{~kg} / \mathrm{hl}$ for 'C 591 and WH 800 ' in first year. All the genotypes in tall wheats group gave high values for test weight along with the commercial; wheats and the advanced lines known to have good grains. Only PBW 534 gave a value of $76.67 \mathrm{~kg} / \mathrm{hl}$. In second year the values of test weight ranged from 73.50 $\mathrm{kg} / \mathrm{hl}$ for 'Lok 1' to $79.33 \mathrm{~kg} / \mathrm{hl}$ for 'C 591'. Overall the values of test weight observed were on lower side in comparison to previous season. The 1000 grain weight varied from $27.18 \mathrm{~g}$ for 'DI 9' to 42.54 $\mathrm{g}$ for 'KYZ-K2K-13' in first year. The advanced lines with good grains and the commercial varieties gave high 1000 grain weight values in comparison to tall wheats which gave medium range of values for this trait. All the genetic stocks with an exception of KYZ-K2K-13 (42.54 g) and WH 800 $(39.31 \mathrm{~g})$ gave low to medium range of values. Glupro however gave a very low value of $21.14 \mathrm{~g}$ for 1000 grain weight. The range was from $36.75 \mathrm{~g}$ for 'HI 1418' to $52.07 \mathrm{~g}$ for 'PBW 533' in second year. The yellow berry incidence may not be directly related with chapati quality but lower incidences are favored as it improves grain hardness. Yellow berry incidence was observed to range from $1.17 \%$ for 'PBW 550 ' to $11.40 \%$ for 'WH 595' in the season first year, where as the range was from $0.93 \%$ for ' $9 \mathrm{D}$ ' to $8.20 \%$ for 'WH 1003 during second year. The tall wheats exhibited lower levels of grain mottling, which may be due to their grains being harder and containing higher protein content than other genotypes. Grain hardness of the genotypes ranged from $10.31 \mathrm{~kg}$ for 'HD 2793' to $14.95 \mathrm{~kg}$ for 'C 591' in first year. In second year the range for hardness was observed 
Satish Kumar et al. / J. Appl. \& Nat. Sci. 10 (2): 572 - 592 (2018)

Table 6. Mean performance of lines for physico-chemical characteristics of ' $A$ ' population.

\begin{tabular}{|c|c|c|c|c|c|c|c|c|c|c|c|c|c|c|c|c|}
\hline Entry & GH & GA & PS & FN & TGW & TW & PC & MC & SV & DP & RS & TS & GW & GD & GI & CS \\
\hline 1 & 8.56 & 6.03 & 4.23 & 432.33 & 45.65 & 78.33 & 14.50 & 10.62 & 55.00 & 192.33 & 0.39 & 3.28 & 27.19 & 8.75 & 47.92 & 7.6 \\
\hline 2 & 8.35 & 5.97 & 4.00 & 465.33 & 45.85 & 78.50 & 14.06 & 10.46 & 59.00 & 213.67 & 0.38 & 3.25 & 30.75 & 9.86 & 46.60 & 7.7 \\
\hline 3 & 8.21 & 5.87 & 3.33 & 446.67 & 40.82 & 77.33 & 13.48 & 10.90 & 47.33 & 192.67 & 0.41 & 2.88 & 25.93 & 8.38 & 43.17 & 7.6 \\
\hline 4 & 7.97 & 5.90 & 3.93 & 489.33 & 47.47 & 76.67 & 13.92 & 10.96 & 61.33 & 231.67 & 0.46 & 3.14 & 31.99 & 10.15 & 52.10 & 7.7 \\
\hline 5 & 8.91 & 5.90 & 4.27 & 493.00 & 50.10 & 77.33 & 13.74 & 10.41 & 57.33 & 187.33 & 0.47 & 3.25 & 28.53 & 9.20 & 60.12 & 7.7 \\
\hline 6 & 8.61 & 5.87 & 4.03 & 469.33 & 43.53 & 77.33 & 13.33 & 10.77 & 62.00 & 225.67 & 0.44 & 3.05 & 28.83 & 9.19 & 44.30 & 7.6 \\
\hline 7 & 8.87 & 5.83 & 4.13 & 508.33 & 39.18 & 78.17 & 13.46 & 10.37 & 72.67 & 253.00 & 0.39 & 3.26 & 26.99 & 9.30 & 47.37 & 7.5 \\
\hline 8 & 8.47 & 5.93 & 4.10 & 521.33 & 42.17 & 79.33 & 13.65 & 9.98 & 62.00 & 263.67 & 0.36 & 2.96 & 30.10 & 10.08 & 36.58 & 7.7 \\
\hline 9 & 8.25 & 5.77 & 4.13 & 447.67 & 36.37 & 76.50 & 13.07 & 10.26 & 55.67 & 227.00 & 0.43 & 3.12 & 15.93 & 5.65 & 69.35 & 7.6 \\
\hline 10 & 11.33 & 5.67 & 4.20 & 397.67 & 48.27 & 76.67 & 14.94 & 11.08 & 57.00 & 200.67 & 0.49 & 3.01 & 34.49 & 11.56 & 36.72 & 7.2 \\
\hline 11 & 9.47 & 5.60 & 4.67 & 529.67 & 41.60 & 76.83 & 13.58 & 10.52 & 66.00 & 242.00 & 0.48 & 3.16 & 25.33 & 8.35 & 55.17 & 7.6 \\
\hline 12 & 10.98 & 5.67 & 3.87 & 422.67 & 40.72 & 77.33 & 14.11 & 10.72 & 60.67 & 212.33 & 0.54 & 3.31 & 29.77 & 10.25 & 23.17 & 7.8 \\
\hline 13 & 8.88 & 5.93 & 4.30 & 405.33 & 40.03 & 78.17 & 14.18 & 10.67 & 59.67 & 181.67 & 0.51 & 3.38 & 29.77 & 9.69 & 38.46 & 7.6 \\
\hline 14 & 7.61 & 5.77 & 4.87 & 362.67 & 44.10 & 78.50 & 15.27 & 10.50 & 55.33 & 164.00 & 0.46 & 3.47 & 32.42 & 10.45 & 46.59 & 7.3 \\
\hline 15 & 8.28 & 5.80 & 4.60 & 391.33 & 34.23 & 75.33 & 14.47 & 10.80 & 52.67 & 173.33 & 0.48 & 3.19 & 33.19 & 9.65 & 47.88 & 7.6 \\
\hline 16 & 8.26 & 5.67 & 4.03 & 414.67 & 45.48 & 79.67 & 16.73 & 10.38 & 57.00 & 219.00 & 0.47 & 3.05 & 41.39 & 13.65 & 31.61 & 7.5 \\
\hline 17 & 7.22 & 5.90 & 4.40 & 428.67 & 40.82 & 78.00 & 15.17 & 10.52 & 54.33 & 194.67 & 0.46 & 3.01 & 32.58 & 10.42 & 51.91 & 7.6 \\
\hline 18 & 7.89 & 5.90 & 3.93 & 435.00 & 36.23 & 79.00 & 15.38 & 10.37 & 52.00 & 227.00 & 0.49 & 3.22 & 37.38 & 12.02 & 28.55 & 7.7 \\
\hline 19 & 8.97 & 5.77 & 4.03 & 475.33 & 35.85 & 76.67 & 14.99 & 10.25 & 58.00 & 206.33 & 0.46 & 3.27 & 32.40 & 10.48 & 71.94 & 7.4 \\
\hline 20 & 6.75 & 6.00 & 0.93 & 473.33 & 33.23 & 78.17 & 14.51 & 10.60 & 70.33 & 259.67 & 0.44 & 3.17 & 34.23 & 11.16 & 23.45 & 7.5 \\
\hline 21 & 8.22 & 5.90 & 4.13 & 345.00 & 39.17 & 79.83 & 16.12 & 10.28 & 63.00 & 194.00 & 0.48 & 3.17 & 35.99 & 11.71 & 78.56 & 7.7 \\
\hline 22 & 9.43 & 5.90 & 4.03 & 420.00 & 43.25 & 79.83 & 15.36 & 10.69 & 66.67 & 214.67 & 0.43 & 2.99 & 36.68 & 12.52 & 59.99 & 7.7 \\
\hline 23 & 9.48 & 5.87 & 3.77 & 386.00 & 43.87 & 80.50 & 15.65 & 10.30 & 63.00 & 174.33 & 0.41 & 3.10 & 35.22 & 11.62 & 83.36 & 7.9 \\
\hline 24 & 9.65 & 5.93 & 3.90 & 468.33 & 39.43 & 78.50 & 14.70 & 10.85 & 59.33 & 235.33 & 0.46 & 3.25 & 29.84 & 10.34 & 65.50 & 7.5 \\
\hline 25 & 11.33 & 6.00 & 4.17 & 405.67 & 41.00 & 81.67 & 16.04 & 10.46 & 65.00 & 227.00 & 0.39 & 3.10 & 34.47 & 11.64 & 72.79 & 7.6 \\
\hline 26 & 9.63 & 5.93 & 2.57 & 504.67 & 50.08 & 78.17 & 14.60 & 10.33 & 57.33 & 242.67 & 0.41 & 3.55 & 31.54 & 10.36 & 7.03 & 7.6 \\
\hline 27 & 9.19 & 5.77 & 3.03 & 515.67 & 44.68 & 76.67 & 13.75 & 10.16 & 48.67 & 219.00 & 0.44 & 3.44 & 29.58 & 9.53 & 41.52 & 7.7 \\
\hline 28 & 8.50 & 5.83 & 0.83 & 497.33 & 39.77 & 75.67 & 13.03 & 10.21 & 47.67 & 231.00 & 0.41 & 3.50 & 29.81 & 9.86 & 27.62 & 7.5 \\
\hline 29 & 9.99 & 5.87 & 2.53 & 485.00 & 50.73 & 77.17 & 13.30 & 10.61 & 50.67 & 200.67 & 0.49 & 3.54 & 28.68 & 8.69 & 51.09 & 7.7 \\
\hline 30 & 9.24 & 5.97 & 1.63 & 435.00 & 44.82 & 77.83 & 13.61 & 10.92 & 49.00 & 176.33 & 0.43 & 3.53 & 25.64 & 8.42 & 33.74 & 7.5 \\
\hline 31 & 9.04 & 5.90 & 1.97 & 437.67 & 36.05 & 76.50 & 13.12 & 10.50 & 50.00 & 229.00 & 0.43 & 3.31 & 27.50 & 9.11 & 52.65 & 7.6 \\
\hline 32 & 8.46 & 6.00 & 2.60 & 524.33 & 38.39 & 78.33 & 13.56 & 10.70 & 56.33 & 246.67 & 0.44 & 3.26 & 29.39 & 9.98 & 36.03 & 7.6 \\
\hline 33 & 7.23 & 6.00 & 2.40 & 467.00 & 38.88 & 79.33 & 13.93 & 10.25 & 58.67 & 250.00 & 0.41 & 3.20 & 30.69 & 10.34 & 18.70 & 7.5 \\
\hline 34 & 9.56 & 5.97 & 3.07 & 460.67 & 40.73 & 77.83 & 13.02 & 10.35 & 47.33 & 216.67 & 0.43 & 3.52 & 27.20 & 8.71 & 45.66 & 7.4 \\
\hline 35 & 8.98 & 5.93 & 3.33 & 475.33 & 38.33 & 78.33 & 13.56 & 10.45 & 59.33 & 186.67 & 0.46 & 3.31 & 28.87 & 9.47 & 41.34 & 7.5 \\
\hline 36 & 5.63 & 5.23 & 3.93 & 476.00 & 29.58 & 67.50 & 13.32 & 10.81 & 57.00 & 236.00 & 0.43 & 3.22 & 26.98 & 8.66 & 82.66 & 7.4 \\
\hline 37 & 7.63 & 5.30 & 4.20 & 510.33 & 30.45 & 68.33 & 12.87 & 10.45 & 53.33 & 252.67 & 0.34 & 3.23 & 19.40 & 6.31 & 55.81 & 7.5 \\
\hline 38 & 6.99 & 5.67 & 4.43 & 524.33 & 39.05 & 74.83 & 12.81 & 10.37 & 53.67 & 238.00 & 0.43 & 3.49 & 25.62 & 8.35 & 65.57 & 7.4 \\
\hline 39 & 9.31 & 5.77 & 4.20 & 452.00 & 39.72 & 75.67 & 11.95 & 10.25 & 52.00 & 238.33 & 0.56 & 3.22 & 16.96 & 5.48 & 74.89 & 7.5 \\
\hline 40 & 6.98 & 5.23 & 4.63 & 501.00 & 56.68 & 69.17 & 13.35 & 10.46 & 68.67 & 246.67 & 0.41 & 3.07 & 29.75 & 9.32 & 68.73 & 7.6 \\
\hline 41 & 8.63 & 5.47 & 3.30 & 494.00 & 33.32 & 72.83 & 13.47 & 10.36 & 58.33 & 232.00 & 0.46 & 2.82 & 25.76 & 8.50 & 48.94 & 7.6 \\
\hline 42 & 7.55 & 6.10 & 1.53 & 477.00 & 45.03 & 79.33 & 13.53 & 10.68 & 50.67 & 181.33 & 0.41 & 2.88 & 29.54 & 9.38 & 48.30 & 7.6 \\
\hline 43 & 8.00 & 5.93 & 3.67 & 566.33 & 43.25 & 77.50 & 13.87 & 10.11 & 51.00 & 208.67 & 0.46 & 3.01 & 29.64 & 9.85 & 29.07 & 7.5 \\
\hline 44 & 6.33 & 5.67 & 3.03 & 494.00 & 35.02 & 74.00 & 12.64 & 10.63 & 54.67 & 221.33 & 0.46 & 2.97 & 18.17 & 5.82 & 83.37 & 7.5 \\
\hline 45 & 8.85 & 6.00 & 3.23 & 448.33 & 41.42 & 78.67 & 13.72 & 10.62 & 60.33 & 194.67 & 0.47 & 3.37 & 33.45 & 10.96 & 32.45 & 7.8 \\
\hline 46 & 8.15 & 5.50 & 3.53 & 535.33 & 30.08 & 70.00 & 12.47 & 10.45 & 59.33 & 186.67 & 0.41 & 2.88 & 14.57 & 4.44 & 88.76 & 7.4 \\
\hline 47 & 8.43 & 5.50 & 4.07 & 452.00 & 32.85 & 71.67 & 12.67 & 10.40 & 56.67 & 211.67 & 0.46 & 3.47 & 21.49 & 6.98 & 62.89 & 7.1 \\
\hline 48 & 8.71 & 5.47 & 4.07 & 501.00 & 31.35 & 71.67 & 12.66 & 10.87 & 57.33 & 237.67 & 0.44 & 3.06 & 23.89 & 7.59 & 86.11 & 7.7 \\
\hline 49 & 7.88 & 5.47 & 4.17 & 493.00 & 30.98 & 70.33 & 13.26 & 10.50 & 57.00 & 237.67 & 0.46 & 3.57 & 28.85 & 9.08 & 62.14 & 7.5 \\
\hline 50 & 6.70 & 5.63 & 4.40 & 516.33 & 33.67 & 72.67 & 13.50 & 10.19 & 59.00 & 241.67 & 0.46 & 3.31 & 24.59 & 7.91 & 78.69 & 7.3 \\
\hline 51 & 7.41 & 5.97 & 3.83 & 500.00 & 35.45 & 76.17 & 12.08 & 10.89 & 53.33 & 253.00 & 0.38 & 2.94 & 26.67 & 8.44 & 47.69 & 7.5 \\
\hline 52 & 6.73 & 4.97 & 1.40 & 494.67 & 26.85 & 66.50 & 12.87 & 10.91 & 51.33 & 218.67 & 0.46 & 2.88 & 24.66 & 7.77 & 66.77 & 7.6 \\
\hline 53 & 7.60 & 5.20 & 1.33 & 491.33 & 26.80 & 68.67 & 12.83 & 10.55 & 48.33 & 205.00 & 0.43 & 2.78 & 18.40 & 6.06 & 57.75 & 7.3 \\
\hline 54 & 8.11 & 5.10 & 2.27 & 475.67 & 26.70 & 66.00 & 12.65 & 10.64 & 52.00 & 189.00 & 0.36 & 3.15 & 18.39 & 4.92 & 69.75 & 7.1 \\
\hline 55 & 8.83 & 5.80 & 2.40 & 509.67 & 35.23 & 75.00 & 11.72 & 10.10 & 54.00 & 210.00 & 0.48 & 2.85 & 21.32 & 7.06 & 22.82 & 7.3 \\
\hline 56 & 6.15 & 5.37 & 3.20 & 544.67 & 30.22 & 71.33 & 12.03 & 10.54 & 52.67 & 177.33 & 0.43 & 2.94 & 12.24 & 4.42 & 75.46 & 7.0 \\
\hline 57 & 8.57 & 6.10 & 4.07 & 470.00 & 38.33 & 78.67 & 13.85 & 10.33 & 55.33 & 178.67 & 0.36 & 2.69 & 29.90 & 10.05 & 38.55 & 7.2 \\
\hline 58 & 8.02 & 6.13 & 4.27 & 470.67 & 41.55 & 78.33 & 13.99 & 10.68 & 59.00 & 181.67 & 0.43 & 3.02 & 29.76 & 9.48 & 52.74 & 7.3 \\
\hline 59 & 9.21 & 5.67 & 4.43 & 494.00 & 36.03 & 74.67 & 13.13 & 10.63 & 53.00 & 210.67 & 0.36 & 3.33 & 24.90 & 8.32 & 48.45 & 7.5 \\
\hline 60 & 9.67 & 5.80 & 2.80 & 561.67 & 43.72 & 74.67 & 12.93 & 10.30 & 55.67 & 193.00 & 0.51 & 3.34 & 24.63 & 7.62 & 72.62 & 7.6 \\
\hline 61 & 9.34 & 6.03 & 3.87 & 503.00 & 45.20 & 79.50 & 14.42 & 10.59 & 56.00 & 253.00 & 0.44 & 3.18 & 33.95 & 10.85 & 38.49 & 7.5 \\
\hline 62 & 7.74 & 5.50 & 4.03 & 469.00 & 45.08 & 77.67 & 13.69 & 10.48 & 54.33 & 179.00 & 0.44 & 3.77 & 30.27 & 9.65 & 44.74 & 7.4 \\
\hline 63 & 7.13 & 5.43 & 4.17 & 371.67 & 48.63 & 78.50 & 12.66 & 10.45 & 45.67 & 139.00 & 0.46 & 3.69 & 29.60 & 9.63 & 54.74 & 7.7 \\
\hline 64 & 8.47 & 5.50 & 3.93 & 451.33 & 44.60 & 77.83 & 13.70 & 10.19 & 51.67 & 210.67 & 0.51 & 3.61 & 30.77 & 10.19 & 37.12 & 7.5 \\
\hline 65 & 7.11 & 5.93 & 4.20 & 503.00 & 41.70 & 78.50 & 12.43 & 10.29 & 56.33 & 211.00 & 0.46 & 3.57 & 26.55 & 8.39 & 55.37 & 7.6 \\
\hline 66 & 8.75 & 5.73 & 4.40 & 500.00 & 38.38 & 75.17 & 11.90 & 10.36 & 53.67 & 228.00 & 0.43 & 2.67 & 23.33 & 7.44 & 46.41 & 7.5 \\
\hline 67 & 10.49 & 5.83 & 4.37 & 579.33 & 42.73 & 79.00 & 12.47 & 10.80 & 51.00 & 252.67 & 0.48 & 3.04 & 27.05 & 8.63 & 52.05 & 7.5 \\
\hline 68 & 10.81 & 5.77 & 4.47 & 440.33 & 41.07 & 80.50 & 14.66 & 9.92 & 56.33 & 184.33 & 0.48 & 2.88 & 30.90 & 10.39 & 57.58 & 7.3 \\
\hline
\end{tabular}


Satish Kumar et al. / J. Appl. \& Nat. Sci. 10 (2): 572 - 592 (2018)

\begin{tabular}{|c|c|c|c|c|c|c|c|c|c|c|c|c|c|c|c|c|}
\hline 69 & 10.04 & 5.67 & 4.47 & 514.33 & 32.75 & 74.33 & 12.86 & 9.88 & 55.00 & 270.00 & 0.49 & 2.99 & 27.08 & 8.68 & 41.15 & 7.5 \\
\hline 70 & 8.11 & 6.03 & 4.07 & 546.33 & 36.10 & 77.67 & 13.49 & 9.86 & 55.67 & 219.00 & 0.51 & 2.90 & 23.78 & 8.05 & 53.69 & 7.5 \\
\hline $\begin{array}{l}\text { C } 273 \\
\text { PBW }\end{array}$ & 12.36 & 5.93 & 1.87 & 584.00 & 37.90 & 76.83 & 12.97 & 9.20 & 53.00 & 368.00 & 0.54 & 3.40 & 32.80 & 10.30 & 41.78 & 8.2 \\
\hline $\begin{array}{l}343 \\
\text { CD }\end{array}$ & 9.52 & 5.50 & 6.90 & 600.00 & 48.56 & 75.00 & 11.22 & 9.16 & 34.67 & 379.33 & 0.56 & 2.93 & 26.87 & 7.90 & 40.26 & 7.7 \\
\hline$(5 \%)$ & 1.94 & 0.35 & 0.88 & 75.79 & 8.58 & 1.85 & 1.00 & 0.61 & 11.02 & 50.56 & ns & 0.42 & 7.00 & 2.12 & 19.99 & \\
\hline
\end{tabular}

$\mathrm{GA}=$ Grain appearance (out of 10$), \mathrm{TGW}=1000$ grain weight $(\mathrm{g}), \mathrm{TW}=$ Test weight $(\mathrm{kh} / \mathrm{hl}), \mathrm{YB}=$ Yellow berry $(\%), \mathrm{GH}=$ Grain hardness $(\mathrm{kg}), \quad \mathrm{PS}=$ phenol score (out of 10$), \quad \mathrm{MC}=$ moisture content $(\%), \mathrm{PC}=$ Protein content $(\%), \mathrm{SV}=$ Sedimentation value $(\mathrm{cc}), \mathrm{DP}=$ Diastatic power $(\mathrm{mg}), \mathrm{FN}=$ Falling number, $\mathrm{CC}=$ Carotenoids $(\mathrm{ppm}), \mathrm{RS}=$ Reducing sugars $(\%), \mathrm{TS}=$ Total sugars, $\mathrm{GW}=$ wet gluten $(\%), \mathrm{GD}=$ Dry gluten, $\mathrm{GI}=$ Gluten index, $\mathrm{CS}=$ chapati score (out of 10).

Table 7. Mean performance of lines for physico-chemical characteristics of 'B' population.

\begin{tabular}{|c|c|c|c|c|c|c|c|c|c|c|c|c|c|c|c|c|}
\hline Entry & $\mathrm{GH}$ & GA & PS & $\mathrm{FN}$ & TGW & TW & $\mathrm{PC}$ & MC & SV & DP & RS & TS & GW & GD & GI & CS \\
\hline 1 & 10.15 & 6.17 & 3.73 & 425.33 & 40.95 & 80.00 & 14.30 & 10.97 & 55.67 & 261.00 & 0.61 & 2.00 & 22.58 & 7.86 & 82.52 & 7.7 \\
\hline 2 & 8.53 & 6.07 & 3.80 & 497.67 & 37.78 & 78.67 & 14.11 & 10.54 & 61.33 & 272.67 & 0.49 & 1.99 & 27.84 & 9.71 & 56.60 & 8.0 \\
\hline 3 & 9.93 & 6.17 & 3.87 & 469.67 & 42.30 & 80.00 & 13.28 & 10.95 & 59.00 & 277.00 & 0.45 & 1.84 & 23.96 & 7.05 & 84.41 & 7.9 \\
\hline 4 & 8.39 & 4.80 & 4.13 & 469.00 & 33.83 & 79.33 & 15.09 & 10.49 & 56.00 & 265.67 & 0.44 & 2.04 & 32.14 & 10.50 & 48.10 & 7.9 \\
\hline 5 & 11.23 & 5.57 & 4.60 & 579.67 & 47.00 & 78.17 & 15.29 & 10.77 & 51.00 & 290.00 & 0.39 & 1.60 & 34.67 & 10.91 & 58.02 & 7.9 \\
\hline 6 & 10.11 & 5.80 & 4.60 & 406.67 & 43.93 & 79.67 & 14.15 & 10.96 & 56.33 & 210.00 & 0.54 & 1.74 & 28.28 & 9.61 & 68.44 & 7.9 \\
\hline 7 & 10.05 & 6.00 & 4.27 & 536.67 & 41.90 & 79.00 & 13.95 & 10.58 & 57.33 & 250.33 & 0.48 & 1.78 & 27.83 & 8.04 & 90.76 & 7.4 \\
\hline 8 & 8.95 & 5.97 & 4.10 & 497.33 & 40.88 & 76.67 & 14.13 & 11.21 & 54.67 & 272.33 & 0.53 & 1.89 & 25.00 & 9.03 & 74.73 & 7.7 \\
\hline 9 & 8.96 & 5.73 & 4.20 & 480.67 & 43.08 & 79.17 & 15.69 & 10.98 & 56.00 & 297.00 & 0.59 & 1.70 & 34.28 & 11.33 & 84.14 & 7.6 \\
\hline 10 & 10.85 & 5.80 & 3.13 & 526.00 & 41.85 & 77.50 & 16.22 & 10.54 & 56.67 & 280.33 & 0.52 & 1.68 & 38.45 & 13.12 & 49.78 & 7.8 \\
\hline 11 & 10.51 & 5.87 & 3.73 & 531.33 & 46.32 & 77.33 & 15.79 & 11.05 & 59.33 & 323.67 & 0.51 & 1.66 & 36.05 & 12.15 & 71.28 & 7.9 \\
\hline 12 & 9.26 & 5.53 & 4.50 & 501.67 & 36.28 & 74.67 & 15.20 & 11.03 & 54.33 & 288.67 & 0.56 & 1.81 & 33.01 & 11.51 & 70.61 & 7.7 \\
\hline 13 & 10.04 & 5.33 & 4.53 & 474.00 & 35.72 & 73.17 & 14.65 & 10.67 & 56.67 & 252.33 & 0.48 & 1.78 & 29.03 & 9.99 & 86.14 & 7.9 \\
\hline 14 & 8.55 & 4.70 & 4.30 & 470.67 & 33.80 & 72.83 & 15.94 & 10.74 & 53.00 & 284.67 & 0.82 & 1.61 & 33.44 & 11.42 & 71.23 & 7.8 \\
\hline 15 & 9.13 & 6.03 & 4.40 & 486.67 & 41.32 & 77.00 & 14.56 & 10.88 & 62.67 & 258.67 & 0.64 & 1.83 & 30.81 & 10.46 & 79.84 & 7.9 \\
\hline 16 & 9.04 & 5.80 & 4.23 & 537.00 & 39.75 & 76.67 & 13.38 & 10.57 & 53.00 & 291.33 & 0.49 & 1.87 & 19.48 & 7.06 & 78.76 & 7.9 \\
\hline 17 & 10.61 & 5.60 & 4.20 & 414.67 & 35.83 & 73.83 & 15.82 & 10.72 & 54.67 & 261.33 & 0.54 & 1.82 & 34.44 & 11.57 & 80.99 & 7.9 \\
\hline 18 & 10.19 & 4.13 & 3.87 & 427.00 & 33.23 & 70.67 & 16.79 & 11.02 & 64.00 & 280.33 & 0.51 & 1.76 & 39.36 & 13.50 & 43.22 & 7.8 \\
\hline 19 & 10.90 & 5.80 & 3.53 & 524.33 & 43.90 & 78.67 & 12.90 & 10.96 & 50.67 & 314.33 & 0.53 & 1.84 & 21.72 & 6.31 & 85.03 & 7.7 \\
\hline 20 & 8.73 & 5.60 & 4.07 & 399.33 & 39.95 & 74.00 & 15.75 & 10.87 & 54.33 & 304.33 & 0.59 & 1.77 & 36.24 & 12.39 & 54.15 & 8.0 \\
\hline 21 & 10.11 & 5.53 & 3.53 & 414.67 & 41.53 & 77.33 & 15.12 & 10.69 & 54.00 & 232.00 & 0.54 & 1.97 & 29.66 & 10.09 & 81.81 & 7.6 \\
\hline 22 & 11.16 & 5.73 & 3.93 & 496.67 & 42.00 & 79.00 & 14.31 & 10.91 & 55.33 & 246.67 & 0.56 & 1.79 & 30.27 & 10.26 & 71.59 & 7.5 \\
\hline 23 & 9.64 & 5.90 & 4.30 & 438.33 & 42.40 & 80.17 & 14.83 & 10.81 & 52.67 & 236.00 & 0.52 & 1.76 & 30.81 & 10.28 & 80.71 & 7.4 \\
\hline 24 & 10.61 & 5.97 & 2.87 & 434.00 & 42.95 & 79.17 & 14.52 & 10.89 & 54.67 & 250.00 & 0.56 & 2.01 & 31.92 & 10.48 & 54.42 & 7.0 \\
\hline 25 & 9.75 & 5.93 & 3.27 & 500.33 & 41.00 & 79.83 & 14.70 & 11.27 & 57.67 & 274.00 & 0.49 & 1.73 & 35.77 & 11.73 & 71.19 & 7.2 \\
\hline 26 & 8.64 & 5.97 & 3.67 & 538.67 & 41.12 & 79.17 & 15.03 & 10.80 & 62.67 & 288.67 & 0.54 & 1.69 & 34.52 & 11.77 & 64.21 & 7.0 \\
\hline 27 & 7.56 & 5.93 & 3.80 & 566.33 & 41.10 & 78.67 & 14.08 & 10.77 & 60.67 & 289.00 & 0.48 & 1.69 & 33.43 & 10.96 & 73.49 & 7.2 \\
\hline 28 & 9.08 & 5.77 & 3.47 & 551.33 & 40.90 & 77.67 & 14.83 & 11.06 & 60.67 & 287.00 & 0.54 & 1.76 & 38.81 & 13.24 & 61.86 & 7.2 \\
\hline 29 & 7.25 & 3.67 & 3.60 & 458.33 & 27.32 & 64.83 & 15.36 & 10.82 & 48.33 & 198.67 & 0.62 & 1.92 & 23.24 & 7.61 & 81.57 & 7.0 \\
\hline 30 & 7.89 & 3.90 & 3.97 & 557.33 & 24.48 & 66.67 & 12.85 & 10.87 & 48.33 & 302.00 & 0.69 & 1.84 & 16.08 & 4.62 & 85.79 & 6.9 \\
\hline 31 & 9.37 & 6.10 & 3.90 & 540.33 & 39.93 & 78.83 & 15.29 & 10.93 & 63.67 & 244.67 & 0.57 & 2.08 & 33.04 & 11.57 & 84.39 & 7.0 \\
\hline 32 & 10.66 & 6.00 & 3.90 & 497.67 & 39.98 & 78.83 & 13.92 & 11.17 & 63.33 & 291.33 & 0.48 & 2.09 & 28.14 & 10.00 & 89.78 & 7.3 \\
\hline 33 & 11.95 & 5.83 & 3.97 & 486.67 & 37.45 & 75.83 & 13.43 & 11.02 & 55.33 & 263.33 & 0.49 & 2.04 & 24.76 & 7.53 & 89.53 & 7.2 \\
\hline 34 & 10.76 & 6.27 & 3.80 & 477.00 & 43.38 & 79.83 & 14.84 & 10.70 & 57.00 & 257.33 & 0.51 & 2.14 & 30.74 & 10.76 & 80.97 & 7.2 \\
\hline 35 & 8.65 & 5.60 & 3.57 & 510.00 & 34.19 & 73.50 & 12.88 & 11.06 & 57.33 & 234.00 & 0.48 & 1.98 & 28.76 & 8.66 & 89.86 & 7.1 \\
\hline 36 & 5.56 & 2.40 & 3.60 & 438.67 & 21.23 & 60.00 & 15.20 & 11.15 & 54.00 & 263.33 & 0.49 & 1.85 & 31.23 & 10.63 & 89.89 & 7.3 \\
\hline 37 & 8.67 & 4.47 & 3.83 & 496.00 & 31.67 & 67.83 & 13.84 & 11.16 & 55.67 & 250.00 & 0.53 & 1.74 & 30.94 & 9.30 & 77.16 & 6.9 \\
\hline 38 & 8.28 & 4.00 & 3.60 & 460.33 & 30.37 & 66.33 & 14.76 & 10.97 & 51.33 & 248.67 & 0.49 & 1.58 & 28.61 & 9.53 & 73.77 & 6.9 \\
\hline 39 & 7.85 & 2.60 & 3.23 & 400.33 & 24.97 & 59.50 & 15.50 & 10.98 & 52.67 & 263.00 & 0.43 & 1.92 & 31.58 & 10.40 & 87.88 & 6.9 \\
\hline 40 & 10.45 & 5.70 & 3.93 & 477.67 & 44.05 & 79.00 & 15.44 & 10.52 & 58.33 & 275.67 & 0.44 & 1.65 & 31.79 & 11.21 & 77.92 & 7.1 \\
\hline 41 & 10.29 & 3.07 & 3.33 & 464.00 & 27.85 & 64.67 & 14.55 & 11.11 & 53.00 & 250.33 & 0.53 & 1.79 & 27.46 & 9.40 & 89.31 & 7.3 \\
\hline 42 & 8.27 & 2.60 & 3.73 & 451.00 & 19.58 & 58.50 & 15.75 & 10.94 & 54.33 & 244.33 & 0.51 & 1.95 & 29.86 & 9.88 & 89.37 & 7.2 \\
\hline 43 & 7.17 & 3.97 & 3.70 & 448.33 & 25.60 & 63.83 & 14.99 & 11.03 & 58.67 & 243.33 & 0.57 & 1.81 & 30.97 & 8.96 & 74.52 & 7.0 \\
\hline 44 & 9.23 & 4.53 & 4.10 & 471.67 & 32.77 & 70.83 & 14.81 & 11.07 & 58.67 & 219.33 & 0.56 & 1.77 & 31.13 & 10.80 & 78.62 & 7.2 \\
\hline 45 & 8.83 & 5.70 & 1.43 & 531.67 & 36.35 & 73.83 & 12.44 & 10.90 & 55.00 & 196.33 & 0.44 & 1.96 & 23.00 & 13.00 & 56.33 & 7.0 \\
\hline 46 & 7.13 & 2.80 & 3.63 & 420.67 & 26.63 & 74.00 & 15.76 & 11.02 & 53.33 & 214.67 & 0.54 & 1.96 & 31.60 & 10.39 & 66.31 & 7.3 \\
\hline 47 & 9.85 & 5.93 & 2.67 & 491.00 & 37.78 & 76.17 & 14.65 & 10.72 & 56.33 & 252.67 & 0.51 & 1.95 & 25.71 & 8.98 & 81.91 & 7.1 \\
\hline 48 & 8.45 & 5.83 & 2.60 & 500.33 & 35.40 & 75.83 & 14.16 & 11.12 & 51.33 & 265.00 & 0.51 & 2.26 & 21.02 & 7.49 & 81.28 & 7.4 \\
\hline 49 & 9.62 & 5.83 & 3.40 & 522.67 & 40.98 & 78.00 & 16.01 & 10.56 & 55.00 & 319.00 & 0.46 & 2.00 & 36.02 & 12.47 & 63.30 & 7.3 \\
\hline 50 & 8.24 & 5.80 & 3.83 & 504.00 & 43.27 & 78.67 & 14.25 & 10.68 & 59.67 & 308.33 & 0.46 & 2.17 & 34.07 & 11.87 & 59.19 & 7.5 \\
\hline 51 & 7.06 & 3.10 & 3.43 & 428.67 & 23.53 & 56.00 & 14.97 & 11.30 & 56.67 & 219.33 & 0.43 & 2.12 & 26.82 & 9.18 & 81.20 & 7.1 \\
\hline 52 & 6.45 & 2.07 & 2.77 & 449.33 & 23.45 & 62.83 & 16.00 & 10.95 & 56.67 & 232.00 & 0.49 & 2.12 & 28.19 & 9.31 & 78.00 & 7.0 \\
\hline 53 & 8.77 & 5.50 & 3.37 & 487.00 & 32.23 & 77.17 & 13.32 & 10.88 & 59.67 & 209.33 & 0.49 & 2.25 & 25.35 & 6.75 & 66.47 & 7.4 \\
\hline 54 & 9.28 & 3.33 & 3.63 & 495.67 & 27.18 & 77.67 & 14.00 & 11.39 & 56.67 & 235.33 & 0.49 & 2.33 & 23.93 & 8.06 & 88.87 & 7.0 \\
\hline 55 & 9.68 & 4.77 & 3.17 & 533.67 & 36.12 & 73.67 & 13.69 & 10.97 & 53.00 & 233.67 & 0.49 & 2.15 & 24.21 & 8.14 & 78.33 & 7.2 \\
\hline 56 & 9.65 & 5.70 & 3.73 & 527.00 & 40.68 & 73.00 & 12.64 & 11.09 & 58.33 & 244.33 & 0.46 & 2.17 & 28.15 & 9.24 & 56.17 & 7.2 \\
\hline 57 & 9.70 & 5.80 & 3.30 & 566.00 & 39.25 & 74.50 & 13.07 & 11.02 & 57.00 & 250.67 & 0.49 & 2.18 & 28.53 & 9.62 & 67.53 & 7.1 \\
\hline
\end{tabular}


Satish Kumar et al. / J. Appl. \& Nat. Sci. 10 (2): 572 - 592 (2018)

\begin{tabular}{|c|c|c|c|c|c|c|c|c|c|c|c|c|c|c|c|c|}
\hline 58 & 7.29 & 1.97 & 3.37 & 464.00 & 20.63 & 57.67 & 14.71 & 11.29 & 56.00 & 237.33 & 0.41 & 2.19 & 26.50 & 7.09 & 91.83 & 7.1 \\
\hline 59 & 6.83 & 4.60 & 3.57 & 535.67 & 30.80 & 67.00 & 14.13 & 10.91 & 55.33 & 244.67 & 0.49 & 2.34 & 33.55 & 8.73 & 74.39 & 7.0 \\
\hline 60 & 12.51 & 5.90 & 4.20 & 358.67 & 43.85 & 79.17 & 14.77 & 10.80 & 63.33 & 206.67 & 0.50 & 2.21 & 31.35 & 9.82 & 78.69 & 7.0 \\
\hline 61 & 8.49 & 2.53 & 3.80 & 436.33 & 25.93 & 65.83 & 16.36 & 10.76 & 48.67 & 250.00 & 0.47 & 2.15 & 37.43 & 12.50 & 78.67 & 7.2 \\
\hline 62 & 7.41 & 3.83 & 3.83 & 471.33 & 27.92 & 66.50 & 15.22 & 10.68 & 51.00 & 248.33 & 0.53 & 2.36 & 33.05 & 10.87 & 59.58 & 7.0 \\
\hline 63 & 7.85 & 4.43 & 3.43 & 480.33 & 31.78 & 67.00 & 13.72 & 11.41 & 51.67 & 250.67 & 0.41 & 2.30 & 31.28 & 10.10 & 77.84 & 7.0 \\
\hline 64 & 9.58 & 5.90 & 3.37 & 520.33 & 35.43 & 76.50 & 13.84 & 11.42 & 59.33 & 237.67 & 0.46 & 2.22 & 31.05 & 10.75 & 71.70 & 6.9 \\
\hline 65 & 10.91 & 5.90 & 3.47 & 428.00 & 43.10 & 79.67 & 14.06 & 11.07 & 54.67 & 226.00 & 0.61 & 2.15 & 28.26 & 9.57 & 79.44 & 7.1 \\
\hline 66 & 9.09 & 3.13 & 3.00 & 481.67 & 21.43 & 62.17 & 15.48 & 11.03 & 50.33 & 242.00 & 0.58 & 2.06 & 25.45 & 8.57 & 79.49 & 7.2 \\
\hline 67 & 6.55 & 3.97 & 3.03 & 447.67 & 23.00 & 61.33 & 14.39 & 11.30 & 55.33 & 252.33 & 0.54 & 1.87 & 30.89 & 10.73 & 82.69 & 7.2 \\
\hline 68 & 7.05 & 2.93 & 3.77 & 419.67 & 24.70 & 60.00 & 13.61 & 11.34 & 57.33 & 169.67 & 0.47 & 1.85 & 31.68 & 9.05 & 72.75 & 7.2 \\
\hline 69 & 10.45 & 5.77 & 3.57 & 274.67 & 47.80 & 76.83 & 13.88 & 11.01 & 59.67 & 138.67 & 0.51 & 1.98 & 26.17 & 9.36 & 83.67 & 7.0 \\
\hline 70 & 7.96 & 2.13 & 3.67 & 417.67 & 21.73 & 59.50 & 14.69 & 11.41 & 46.33 & 244.33 & 0.56 & 2.28 & 25.69 & 8.80 & 46.92 & 7.2 \\
\hline $\begin{array}{l}\text { C } 306 \\
\text { PBW }\end{array}$ & 13.37 & 5.77 & 3.00 & 499.33 & 35.27 & 79.67 & 11.81 & 11.12 & 41.00 & 413.67 & 0.51 & 3.41 & 27.53 & 9.30 & 34.53 & 8.2 \\
\hline 534 & 10.59 & 5.53 & 7.77 & 578.00 & 40.75 & 76.67 & 10.77 & 9.47 & 41.00 & 414.33 & 0.49 & 3.08 & 32.27 & 9.80 & 47.47 & 7.4 \\
\hline CD (5\%) & 2.01 & 1.35 & 0.83 & 90.23 & 9.01 & 6.81 & 1.71 & 0.51 & 9.82 & 70.38 & ns & 0.46 & 7.16 & 2.46 & 19.07 & \\
\hline
\end{tabular}

$\mathrm{GA}=$ Grain appearance (out of 10), TGW = 1000 grain weight $(\mathrm{g}), \mathrm{TW}=$ Test weight $(\mathrm{kh} / \mathrm{hl})$, YB $=$ Yellow berry $(\%)$, GH = Grain hardness $(\mathrm{kg}), \mathrm{PS}=\mathrm{phenol}$ score $($ out of 10$), M C=$ moisture content $(\%), P C=$ Protein content $(\%), S V=$ Sedimentation value $(\mathrm{cc}), \mathrm{DP}=$ Diastatic power $(\mathrm{mg}), \mathrm{FN}=\mathrm{Falling}$ number, $\mathrm{CC}=\mathrm{Carotenoids}(\mathrm{ppm})$, $\mathrm{RS}=$ Reducing sugars $(\%), \mathrm{TS}=$ Total sugars, $\mathrm{GW}=$ wet gluten $(\%), \mathrm{GD}=$ Dry gluten, $\mathrm{Gl}=$ Gluten index, CS = chapati score (out of 10).

Table 8. Mean performance of lines for physico-chemical characteristics of ' $C$ ' population.

\begin{tabular}{|c|c|c|c|c|c|c|c|c|c|c|c|c|c|c|c|c|}
\hline Entry & GH & GA & PS & $\mathrm{FN}$ & TGW & TW & PC & MC & SV & DP & RS & TS & GW & GD & GI & CS \\
\hline 1 & 10.45 & 5.73 & 3.57 & 441.00 & 43.63 & 77.00 & 13.68 & 10.86 & 54.33 & 205.67 & 0.44 & 2.56 & 27.88 & 9.86 & 52.51 & 7.6 \\
\hline 2 & 11.46 & 6.03 & 1.87 & 487.00 & 42.98 & 78.83 & 13.92 & 10.82 & 55.00 & 238.00 & 0.41 & 2.45 & 30.83 & 10.34 & 20.45 & 7.5 \\
\hline 3 & 9.83 & 5.60 & 3.20 & 452.00 & 45.77 & 78.50 & 13.68 & 11.06 & 43.33 & 203.33 & 0.44 & 2.22 & 29.97 & 10.01 & 35.93 & 7.2 \\
\hline 4 & 10.53 & 6.03 & 3.63 & 501.00 & 46.10 & 78.83 & 12.55 & 10.82 & 42.33 & 225.67 & 0.44 & 2.44 & 27.42 & 8.91 & 26.75 & 7.4 \\
\hline 5 & 9.94 & 5.67 & 4.23 & 492.33 & 40.55 & 77.17 & 14.58 & 10.33 & 51.00 & 256.67 & 0.43 & 2.50 & 35.80 & 11.83 & 32.78 & 7.2 \\
\hline 6 & 7.92 & 5.73 & 2.93 & 566.33 & 35.88 & 76.67 & 13.95 & 10.78 & 59.33 & 258.67 & 0.39 & 2.19 & 29.96 & 10.05 & 69.14 & 7.5 \\
\hline 7 & 9.93 & 5.93 & 2.33 & 533.00 & 37.02 & 78.33 & 13.97 & 10.63 & 47.00 & 250.67 & 0.38 & 2.30 & 28.32 & 9.22 & 57.13 & 7.7 \\
\hline 8 & 8.11 & 5.70 & 1.27 & 467.33 & 35.45 & 74.83 & 13.32 & 10.96 & 54.67 & 225.00 & 0.51 & 2.28 & 29.20 & 9.63 & 51.73 & 7.8 \\
\hline 9 & 8.97 & 4.93 & 2.23 & 555.33 & 33.57 & 70.83 & 13.51 & 10.93 & 59.00 & 272.00 & 0.41 & 2.14 & 30.61 & 10.18 & 55.94 & 7.4 \\
\hline 10 & 9.36 & 4.57 & 2.13 & 535.33 & 29.12 & 69.67 & 13.26 & 10.79 & 57.33 & 197.33 & 0.46 & 2.29 & 30.94 & 10.17 & 61.74 & 7.4 \\
\hline 11 & 10.68 & 5.60 & 3.80 & 554.33 & 35.62 & 78.67 & 12.53 & 10.72 & 50.67 & 203.67 & 0.44 & 2.30 & 24.50 & 8.09 & 53.94 & 7.4 \\
\hline 12 & 10.37 & 5.97 & 3.10 & 377.33 & 41.43 & 80.67 & 13.52 & 10.73 & 60.67 & 252.67 & 0.49 & 2.20 & 29.20 & 9.62 & 59.25 & 7.5 \\
\hline 13 & 9.65 & 5.93 & 2.13 & 490.67 & 47.38 & 79.17 & 13.78 & 11.06 & 56.33 & 217.00 & 0.56 & 2.44 & 29.32 & 9.88 & 53.47 & 7.4 \\
\hline 14 & 10.18 & 5.60 & 2.63 & 545.67 & 51.12 & 77.50 & 14.83 & 10.68 & 52.33 & 237.67 & 0.59 & 2.57 & 32.99 & 10.98 & 28.86 & 7.4 \\
\hline 15 & 10.95 & 5.70 & 2.17 & 547.67 & 44.93 & 77.00 & 13.83 & 10.71 & 58.33 & 223.67 & 0.49 & 2.35 & 28.42 & 9.26 & 56.58 & 7.5 \\
\hline 16 & 10.09 & 5.87 & 3.07 & 574.00 & 40.87 & 77.83 & 13.43 & 10.57 & 55.33 & 247.67 & 0.48 & 2.44 & 26.53 & 9.00 & 47.40 & 7.6 \\
\hline 17 & 9.46 & 5.63 & 3.07 & 499.33 & 37.12 & 77.00 & 13.63 & 10.47 & 60.33 & 248.67 & 0.43 & 2.11 & 27.21 & 9.22 & 29.75 & 7.4 \\
\hline 18 & 10.05 & 5.40 & 3.13 & 548.33 & 42.52 & 78.33 & 12.90 & 10.39 & 49.67 & 196.33 & 0.43 & 2.49 & 25.03 & 8.23 & 30.39 & 7.4 \\
\hline 19 & 9.95 & 5.60 & 3.07 & 530.00 & 46.88 & 76.83 & 11.96 & 11.01 & 42.33 & 199.33 & 0.44 & 2.52 & 20.74 & 6.86 & 70.12 & 7.5 \\
\hline 20 & 10.69 & 5.90 & 3.83 & 540.00 & 37.93 & 77.17 & 12.82 & 11.01 & 55.33 & 257.00 & 0.49 & 2.58 & 27.65 & 8.99 & 35.95 & 7.6 \\
\hline 21 & 10.98 & 5.83 & 3.93 & 180.67 & 49.27 & 79.50 & 15.30 & 10.66 & 49.00 & 80.67 & 0.56 & 2.65 & 34.49 & 11.74 & 22.44 & 7.5 \\
\hline 22 & 10.46 & 6.13 & 2.13 & 470.00 & 43.80 & 81.17 & 14.86 & 10.69 & 51.00 & 255.00 & 0.60 & 2.53 & 34.90 & 11.44 & 55.89 & 7.7 \\
\hline 23 & 9.69 & 6.23 & 4.33 & 485.00 & 43.78 & 81.00 & 14.44 & 10.78 & 54.67 & 265.67 & 0.51 & 2.58 & 33.14 & 10.76 & 60.43 & 7.6 \\
\hline 24 & 9.62 & 5.83 & 1.97 & 435.00 & 43.68 & 78.33 & 12.62 & 10.79 & 56.67 & 151.00 & 0.48 & 2.99 & 23.55 & 7.82 & 47.16 & 7.6 \\
\hline 25 & 8.78 & 5.57 & 3.50 & 493.67 & 38.29 & 73.33 & 13.85 & 10.48 & 44.33 & 229.00 & 0.46 & 2.71 & 29.82 & 10.02 & 24.96 & 7.4 \\
\hline 26 & 10.03 & 6.07 & 1.70 & 578.33 & 46.48 & 80.17 & 13.10 & 10.54 & 49.67 & 246.67 & 0.39 & 2.72 & 29.79 & 7.47 & 62.84 & 7.7 \\
\hline 27 & 10.95 & 6.03 & 4.17 & 387.00 & 46.95 & 79.50 & 15.18 & 10.69 & 48.33 & 186.00 & 0.56 & 2.78 & 30.38 & 10.17 & 66.68 & 7.5 \\
\hline 28 & 9.25 & 6.00 & 2.17 & 567.67 & 47.22 & 79.67 & 14.06 & 10.46 & 54.67 & 257.00 & 0.57 & 2.58 & 29.99 & 9.91 & 41.87 & 7.7 \\
\hline 29 & 9.39 & 6.00 & 3.07 & 476.00 & 50.57 & 80.17 & 12.75 & 10.69 & 52.00 & 227.33 & 0.43 & 2.51 & 29.57 & 9.65 & 45.23 & 7.4 \\
\hline 30 & 9.27 & 5.43 & 3.53 & 542.00 & 39.20 & 75.33 & 10.99 & 10.62 & 45.00 & 208.67 & 0.41 & 2.59 & 21.05 & 5.62 & 66.37 & 7.4 \\
\hline 31 & 9.07 & 5.97 & 1.73 & 537.00 & 42.03 & 79.67 & 13.56 & 10.91 & 47.33 & 227.67 & 0.39 & 2.53 & 28.71 & 9.97 & 46.44 & 7.8 \\
\hline 32 & 9.15 & 5.83 & 1.37 & 518.00 & 40.32 & 79.00 & 12.70 & 10.64 & 46.00 & 261.00 & 0.41 & 2.50 & 29.14 & 9.77 & 21.67 & 7.6 \\
\hline 33 & 8.94 & 5.97 & 3.27 & 549.67 & 39.35 & 77.17 & 12.02 & 10.74 & 48.33 & 231.67 & 0.39 & 2.39 & 23.25 & 7.90 & 60.78 & 7.8 \\
\hline 34 & 9.85 & 6.03 & 4.03 & 554.00 & 41.75 & 79.00 & 12.54 & 10.94 & 43.33 & 217.33 & 0.39 & 2.55 & 24.76 & 8.20 & 57.70 & 7.8 \\
\hline 35 & 8.89 & 3.23 & 3.07 & 484.33 & 24.70 & 63.83 & 13.48 & 10.84 & 42.67 & 185.67 & 0.41 & 1.98 & 17.34 & 6.41 & 75.98 & 7.3 \\
\hline 36 & 10.51 & 6.03 & 3.27 & 471.33 & 42.48 & 79.50 & 14.62 & 10.79 & 58.00 & 259.67 & 0.39 & 2.26 & 29.50 & 9.70 & 78.02 & 7.6 \\
\hline 37 & 11.07 & 5.97 & 3.50 & 385.33 & 44.08 & 78.83 & 15.23 & 10.41 & 54.33 & 188.00 & 0.41 & 2.22 & 32.16 & 10.67 & 64.92 & 7.7 \\
\hline 38 & 9.48 & 4.77 & 3.30 & 421.00 & 38.92 & 73.17 & 13.95 & 10.67 & 53.33 & 168.33 & 0.38 & 2.28 & 28.96 & 9.98 & 70.57 & 7.5 \\
\hline 39 & 8.69 & 5.83 & 2.37 & 478.00 & 41.72 & 77.17 & 13.34 & 10.78 & 46.33 & 195.00 & 0.32 & 2.06 & 28.51 & 9.48 & 32.25 & 7.5 \\
\hline 40 & 8.08 & 6.03 & 4.20 & 412.00 & 34.65 & 75.17 & 13.01 & 10.80 & 47.00 & 156.00 & 0.39 & 2.22 & 27.04 & 8.68 & 63.55 & 7.5 \\
\hline 41 & 10.00 & 5.67 & 2.53 & 483.33 & 45.75 & 73.83 & 13.36 & 11.10 & 43.00 & 198.33 & 0.39 & 2.02 & 28.66 & 9.40 & 16.54 & 7.6 \\
\hline 42 & 8.52 & 5.63 & 3.10 & 459.33 & 51.10 & 77.67 & 13.97 & 11.00 & 43.33 & 134.00 & 0.36 & 2.03 & 34.65 & 11.72 & 19.79 & 7.5 \\
\hline 43 & 9.51 & 5.77 & 2.50 & 561.00 & 43.10 & 76.67 & 13.18 & 10.80 & 53.00 & 231.00 & 0.47 & 2.12 & 27.90 & 9.31 & 25.98 & 7.6 \\
\hline 44 & 8.86 & 5.63 & 1.93 & 561.33 & 41.60 & 72.50 & 11.88 & 10.74 & 38.33 & 244.00 & 0.39 & 2.35 & 23.63 & 7.68 & 39.16 & 7.6 \\
\hline 45 & 8.50 & 5.70 & 4.07 & 492.67 & 45.00 & 73.00 & 12.47 & 10.78 & 45.00 & 196.67 & 0.43 & 2.64 & 25.42 & 8.35 & 37.78 & 7.4 \\
\hline 46 & 8.73 & 5.70 & 4.03 & 467.00 & 38.42 & 73.83 & 12.22 & 10.91 & 44.00 & 206.33 & 0.43 & 2.39 & 28.02 & 9.39 & 39.43 & 7.4 \\
\hline 47 & 10.00 & 5.50 & 3.90 & 477.00 & 47.20 & 75.67 & 12.57 & 10.75 & 47.67 & 191.33 & 0.39 & 2.53 & 27.50 & 8.99 & 20.75 & 7.6 \\
\hline 48 & 9.87 & 5.63 & 2.03 & 462.33 & 49.05 & 77.17 & 13.43 & 10.97 & 42.33 & 187.33 & 0.37 & 2.55 & 29.29 & 9.76 & 45.67 & 7.5 \\
\hline 49 & 8.65 & 5.77 & 3.47 & 530.00 & 42.43 & 77.00 & 13.57 & 10.87 & 50.67 & 217.00 & 0.41 & 2.45 & 28.86 & 9.42 & 35.50 & 7.5 \\
\hline 50 & 8.33 & 5.30 & 4.00 & 564.00 & 36.75 & 71.67 & 12.16 & 10.61 & 44.33 & 233.33 & 0.48 & 2.88 & 23.77 & 7.50 & 40.90 & 7.5 \\
\hline
\end{tabular}


Satish Kumar et al. / J. Appl. \& Nat. Sci. 10 (2): 572 - 592 (2018)

\begin{tabular}{|c|c|c|c|c|c|c|c|c|c|c|c|c|c|c|c|c|}
\hline 51 & 9.71 & 5.27 & 3.93 & 546.00 & 37.75 & 71.67 & 12.93 & 10.71 & 50.00 & 271.67 & 0.38 & 2.22 & 28.25 & 9.16 & 54.73 & 7.4 \\
\hline 52 & 10.77 & 5.20 & 3.83 & 514.67 & 41.75 & 75.00 & 12.86 & 10.60 & 44.00 & 227.00 & 0.33 & 2.55 & 24.86 & 7.96 & 55.72 & 7.2 \\
\hline 53 & 8.64 & 5.43 & 2.43 & 516.00 & 30.28 & 70.00 & 11.37 & 10.95 & 38.67 & 220.33 & 0.51 & 2.67 & 17.64 & 5.20 & 78.02 & 7.2 \\
\hline 54 & 7.75 & 5.37 & 1.73 & 521.67 & 32.47 & 69.33 & 13.07 & 11.09 & 47.00 & 223.00 & 0.46 & 2.80 & 28.00 & 8.91 & 69.48 & 7 \\
\hline 55 & 8.39 & 5.73 & 2.33 & 504.67 & 39.68 & 73.50 & 11.82 & 11.12 & 52.33 & 252.00 & 0.46 & 2.75 & 24.98 & 8.18 & 73.49 & 7.3 \\
\hline 56 & 7.96 & 3.27 & 2.33 & 538.67 & 28.33 & 65.67 & 14.49 & 11.17 & 52.33 & 218.67 & 0.41 & 2.75 & 34.28 & 11.61 & 58.80 & 7.4 \\
\hline 57 & 6.69 & 4.03 & 2.20 & 587.00 & 28.23 & 66.00 & 13.31 & 10.87 & 56.00 & 259.00 & 0.41 & 2.39 & 29.14 & 8.87 & 60.46 & 7.5 \\
\hline 58 & 8.12 & 5.37 & 2.50 & 535.33 & 33.35 & 71.17 & 13.27 & 10.66 & 59.67 & 265.67 & 0.39 & 2.71 & 25.77 & 8.67 & 73.46 & 7.5 \\
\hline 59 & 9.45 & 5.13 & 3.13 & 489.00 & 40.55 & 74.83 & 13.51 & 10.75 & 51.00 & 161.00 & 0.41 & 2.62 & 28.67 & 9.26 & 55.14 & 7.2 \\
\hline 60 & 9.82 & 5.43 & 1.97 & 490.33 & 51.17 & 74.17 & 13.41 & 10.94 & 45.33 & 197.67 & 0.44 & 2.38 & 29.40 & 9.40 & 42.62 & 7.2 \\
\hline 61 & 7.55 & 5.10 & 4.43 & 538.67 & 29.38 & 71.33 & 14.53 & 10.59 & 47.33 & 280.00 & 0.41 & 2.12 & 34.86 & 8.49 & 40.04 & 7.5 \\
\hline 62 & 9.38 & 5.33 & 3.40 & 556.67 & 39.25 & 72.17 & 13.49 & 10.94 & 48.67 & 218.67 & 0.49 & 2.13 & 30.58 & 9.98 & 28.89 & 7.3 \\
\hline 63 & 9.81 & 5.87 & 3.73 & 553.00 & 36.75 & 77.83 & 13.43 & 10.40 & 48.67 & 265.33 & 0.52 & 2.39 & 29.46 & 9.41 & 50.69 & 7.3 \\
\hline 64 & 7.85 & 5.43 & 3.67 & 582.00 & 34.20 & 72.83 & 12.43 & 10.55 & 55.67 & 194.67 & 0.42 & 2.37 & 24.78 & 8.31 & 56.22 & 7.1 \\
\hline 65 & 8.58 & 5.77 & 4.13 & 463.67 & 32.38 & 73.17 & 12.24 & 10.85 & 55.00 & 223.33 & 0.37 & 2.25 & 26.00 & 8.51 & 35.31 & 7.4 \\
\hline 66 & 10.00 & 5.00 & 3.97 & 565.00 & 41.49 & 75.30 & 13.42 & 10.60 & 53.00 & 261.00 & 0.53 & 2.44 & 31.36 & 9.57 & 45.36 & 7.3 \\
\hline 67 & 9.71 & 5.83 & 3.97 & 559.33 & 40.67 & 75.33 & 12.66 & 10.78 & 48.33 & 231.67 & 0.46 & 2.33 & 29.66 & 9.54 & 19.06 & 7.4 \\
\hline 68 & 8.67 & 4.23 & 4.17 & 575.67 & 34.65 & 69.00 & 12.45 & 10.49 & 51.00 & 356.00 & 0.38 & 2.25 & 29.13 & 9.07 & 52.51 & 7.1 \\
\hline 69 & 11.54 & 5.40 & 4.17 & 521.00 & 53.33 & 77.00 & 13.39 & 10.77 & 44.00 & 186.67 & 0.38 & 2.44 & 27.22 & 8.89 & 49.21 & 6.8 \\
\hline 70 & 9.23 & 5.63 & 4.57 & 563.67 & 52.67 & 78.00 & 12.11 & 11.08 & 43.33 & 208.67 & 0.39 & 2.52 & 21.85 & 7.13 & 56.53 & 6.9 \\
\hline 71 & 11.09 & 5.67 & 2.93 & 443.67 & 46.80 & 78.33 & 16.23 & 10.73 & 46.67 & 225.67 & 0.46 & 2.29 & 39.13 & 12.46 & 42.60 & 7 \\
\hline 72 & 9.59 & 5.73 & 2.77 & 495.00 & 42.62 & 74.83 & 13.37 & 10.52 & 43.33 & 215.67 & 0.39 & 2.39 & 28.52 & 9.48 & 27.25 & 7.3 \\
\hline 73 & 9.29 & 5.83 & 3.07 & 514.67 & 37.67 & 75.83 & 12.99 & 10.61 & 46.67 & 206.67 & 0.39 & 2.33 & 26.20 & 8.64 & 58.77 & 7.3 \\
\hline 74 & 11.89 & 5.80 & 3.50 & 438.67 & 40.82 & 80.67 & 14.04 & 10.59 & 51.33 & 207.33 & 0.36 & 1.82 & 28.82 & 9.65 & 45.23 & 7.1 \\
\hline 75 & 9.13 & 5.80 & 3.87 & 449.33 & 45.35 & 77.17 & 14.51 & 10.63 & 49.33 & 190.00 & 0.47 & 2.51 & 32.12 & 10.79 & 48.43 & 7.4 \\
\hline 76 & 8.95 & 3.90 & 4.00 & 497.67 & 25.08 & 65.00 & 14.81 & 10.62 & 48.33 & 176.67 & 0.36 & 2.34 & 29.61 & 9.53 & 72.59 & 7.3 \\
\hline 77 & 9.43 & 5.87 & 3.97 & 574.67 & 38.43 & 76.83 & 12.63 & 11.01 & 51.00 & 229.33 & 0.39 & 2.50 & 19.72 & 6.59 & 77.76 & 7.2 \\
\hline 78 & 9.29 & 4.67 & 4.60 & 454.67 & 32.93 & 75.96 & 13.23 & 11.14 & 41.33 & 186.00 & 0.34 & 2.05 & 32.98 & 9.07 & 44.48 & 7.3 \\
\hline 79 & 10.22 & 5.07 & 4.47 & 516.67 & 33.20 & 71.00 & 13.26 & 10.83 & 47.33 & 196.67 & 0.38 & 2.14 & 28.45 & 6.83 & 64.81 & 7.1 \\
\hline 80 & 7.83 & 5.57 & 4.20 & 556.67 & 35.62 & 72.67 & 13.07 & 11.00 & 49.33 & 227.33 & 0.54 & 2.31 & 27.80 & 9.26 & 64.83 & 7.1 \\
\hline $\begin{array}{l}\text { C } 518 \\
\text { PBW }\end{array}$ & 12.31 & 6.17 & 1.67 & 480.67 & 33.71 & 81.33 & 11.95 & 10.75 & 50.67 & 411.67 & 0.51 & 3.40 & 32.90 & 11.37 & 19.95 & $\begin{array}{l}7.9 \\
7.7\end{array}$ \\
\hline $\begin{array}{l}343 \\
\text { CD }\end{array}$ & 10.94 & 5.83 & 5.70 & 578.33 & 40.00 & 79.00 & 10.55 & 10.87 & 38.33 & 456.00 & 0.48 & 3.17 & 24.43 & 7.77 & 50.88 & \\
\hline$(5 \%)$ & 1.77 & 0.87 & 0.81 & 105.26 & 6.18 & 3.90 & 1.34 & ns & 7.74 & 53.25 & 0.14 & 0.55 & 5.67 & 2.14 & 24.85 & \\
\hline
\end{tabular}

$\mathrm{GA}=$ Grain appearance (out of 10), TGW $=1000$ grain weight $(\mathrm{g}), \mathrm{TW}=$ Test weight $(\mathrm{kh} / \mathrm{hl})$, YB $=$ Yellow berry $(\%), \mathrm{GH}=\mathrm{Grain}$ hardness $(\mathrm{kg}), \mathrm{PS}=\mathrm{phenol}$ score $(\mathrm{out}$ of 10$), \mathrm{MC}=$ moisture content $(\%), \mathrm{PC}=$ Protein content $(\%), \mathrm{SV}=$ Sedimentation value (cC), DP = Diastatic power $(\mathrm{mg}), \mathrm{FN}=\mathrm{Falling}$ number, $\mathrm{CC}=\mathrm{Carotenoids}(\mathrm{ppm})$, $\mathrm{RS}=$ Reducing sugars $(\%), \mathrm{TS}=$ Total sugars, $\mathrm{GW}=$ wet gluten $(\%), \mathrm{GD}=$ Dry gluten, $\mathrm{Gl}=$ Gluten index, $\mathrm{CS}=$ chapati score (out of 10 ).

from $9.11 \mathrm{~kg}$ for 'HI 1418 ' to $12.87 \mathrm{~kg}$ for 'C 273 '. The overall trend observed in the genotypes was that the harder genotypes gave higher chapati score. Similarly in second year crop season again the tall varieties were having the hardest grains but Lok 1 earned a high score (7.9/10) in spite of relatively soft grains $(9.4 \mathrm{~kg})$. Phenol score is given on the basis of presence of tyrosinase amount and activity. 'DI 9' showed lowest score of 1.30 and 'PBW 531' gave highest score of 6.00 in first year. A lowest score of 0.87 for 'PBW 175' and a highest score of 8.93 for 'WH 1003' was observed in second year. Lok 1, Central zone wheat known for its good chapati quality gave a high score of 7.03. In case of genetic stocks WH 712 and HD 2793 gave low values over both the seasons.

Grain protein content is considered to be one of the major characters which determine the end use quality of wheat. Higher values of protein content means higher amount of gluten and thus good loaf volume of bread. But for chapati making too high gluten content is not desirable. The tall wheat varieties having good chapati score had about 12 per cent protein content over both the seasons and these wheats recorded the highest values for protein content than other groups in the study. Numerically the protein content was found to vary from $10.21 \%$ for ' $\mathrm{DI} 9$ ' to $12.82 \%$ for 'C 273 ' and from $9.60 \%$ for ' $\mathrm{K} 0123$ ' to $13.14 \%$ for ' $\mathrm{C} 273$ ' in both years. The highest protein content observed in case of tall varieties among the different groups was actually in the medium range desirable for making good chapaties. Glupro, due to its shrivelled grains and presence of $\mathrm{GpcB} 1$ gene gave a protein content of as high as $17.57 \%$.The good chapati wheats were found to have sedimentation value between $40-50 \mathrm{cc}$. The commercial wheats had lower sedimentation value where as the genetic stocks showed a wider range of values from low to very high. The genotypes with higher values could not produce good chapaties, thus indicating the requirement of medium sedimentation values in chapati making. In first year the value of sedimentation was found to range between 38.00 for ' $\mathrm{K} 0123$ ' and 54.67 for 'WH 1003', where as the value ranged between 30.00 for 'PBW 533' and 63.00 for 'WH 1003' in second year. The cultivars known for excellent bread making quality (PBW 533 and DBW 16) had lower sedimentation values, which is in contrast to higher requirements for bread making. The diastatic activity of the genotypes ranged from $319.00 \mathrm{mg}$ for 'Pusa 5-3' to $438.00 \mathrm{mg}$ for 'PBW 531' in first year. In second year the value of diastatic activity varied from $253.00 \mathrm{mg}$ for 'Lok 1' to $496.00 \mathrm{mg}$ for 'HI 1418'. The diastatic power of the genotypes is an indicative of enzymatic activity in the wheat flour. Since harder wheats have more damaged starch, it 


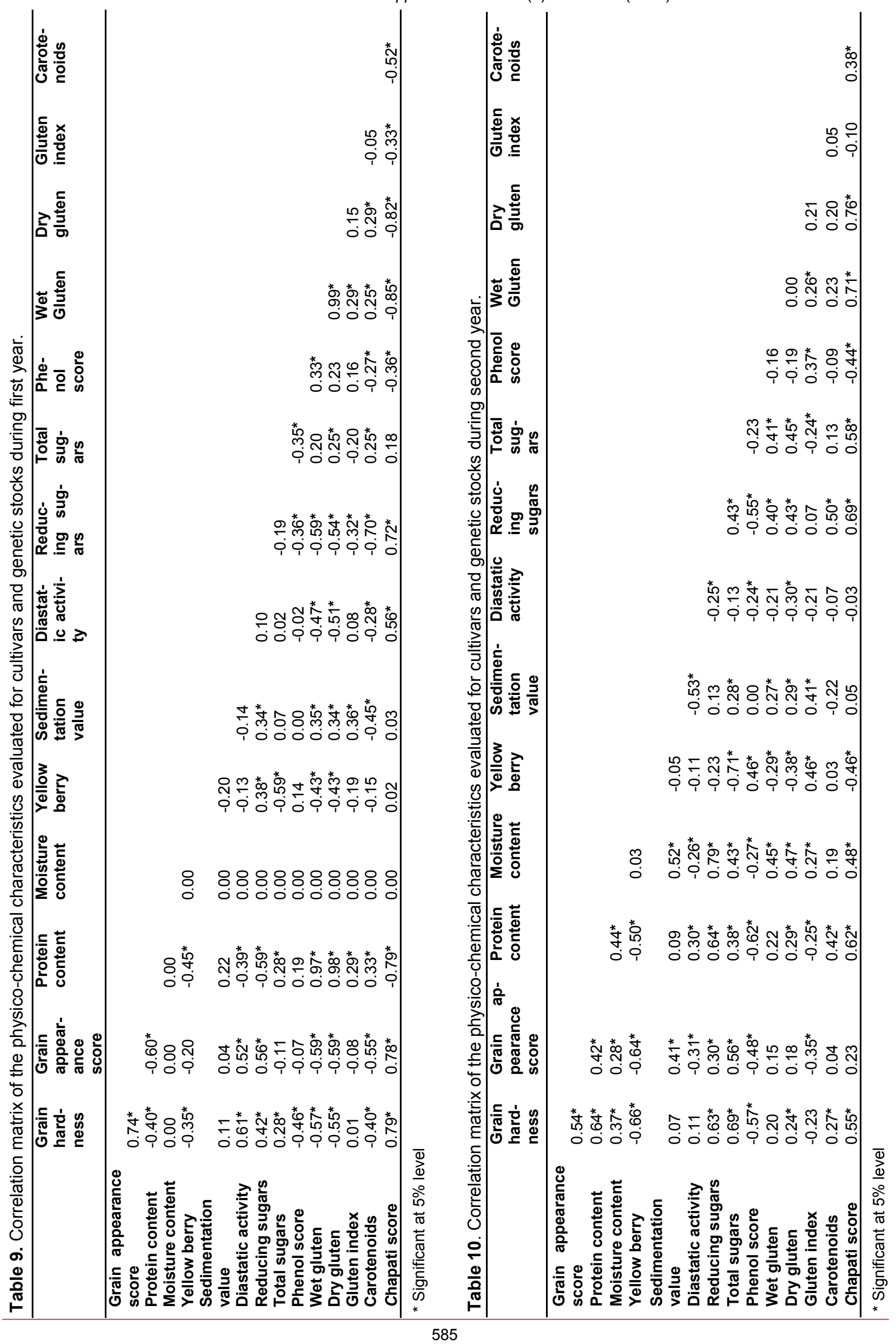


Satish Kumar et al. / J. Appl. \& Nat. Sci. 10 (2): 572 - 592 (2018)

Table 11. Correlation coefficients of the physico-chemical characteristics with chapati score in Back Cross recombinant populations.

\begin{tabular}{llll}
\hline Physico-chemical characteristics & \multicolumn{2}{l}{ Correlation coefficient } \\
\cline { 2 - 4 } & Population A & Population B & Population C \\
\hline Grain hardness & $0.34^{*}$ & $0.35^{*}$ & 0.17 \\
Grain appearance score & $0.26^{*}$ & $0.36^{*}$ & $0.31^{*}$ \\
Protein content & 0.19 & 0.07 & -0.13 \\
Moisture content & -0.19 & $-0.27^{*}$ & -0.02 \\
Sedimentation value & 0.08 & -0.07 & 0.10 \\
Diastatic activity & $0.32^{*}$ & $0.46^{*}$ & $0.41^{*}$ \\
Reducing sugars & $0.25^{*}$ & 0.13 & 0.20 \\
Total sugars & 0.16 & -0.09 & $0.37^{*}$ \\
Phenol score & -0.03 & $0.32^{*}$ & $-0.24^{*}$ \\
Wet gluten & $0.41^{*}$ & 0.12 & 0.06 \\
Dry gluten & 0.39 & 0.14 & 0.11 \\
Gluten index & -0.12 & $-0.34^{*}$ & -0.14 \\
\hline
\end{tabular}

* Significant at $5 \%$ level

gives more sites for the amylases to act, thus increased diastase activity. The values of falling number were found to range between 442.00 for KYZ K2K-13 to 594.33 for PBW 343 in first year. In second year the genotypes in commercial wheats group, genetic stocks HD 2793, WH 712 and $\mathrm{WH} 1003$, the advanced line $\mathrm{HI} 1479$, tall wheats 9D, C 518 and C 591 gave highest value of 600 . HI 1418 gave lowest value of 476.33 . In this season higher values of falling number were observed for all the genotypes. A falling number value in the range of 400 is desirable in good chapati wheats, as a limited activity leads to sweeter taste in chapaties due to the saccharifying activity of the amylases.A value of $3.5 \mathrm{ppm}$ to $4.5 \mathrm{ppm}$ carotenoids is found to impart desirable colour to the chapaties. In first year the value of caretonoids was highest (4.29 ppm) for ' $C$ 591' and lowest (3.00 ppm) for 'PBW 531', where as in second year the values ranged from $3.06 \mathrm{ppm}$ for 'PBW 226 ' to 4.72 ppm for 'PBW 343'. The caretonoids content was highest in the tall varieties and it was 4.15 ppm in C 306, the best chapati quality wheat variety. The total sugars were significantly higher in case of tall wheat varieties thus explaining sweet taste of their chapaties in both the seasons. The total sugar content was observed to vary from 2.8 per cent for 'PBW 502' to 3.4 per cent for ' $C$ 306 ' and ' $\mathrm{C} 518$ ' in first year, where as the value varied from 2.8 per cent for 'PBW 502' to 3.4 per cent for 'C 518' in second year. The commercial wheats had lowest total sugars where as the genetic stocks and advanced lines gave intermediate results. Glupro gave lowest vale of 0.27 per cent reducing sugars. The reducing sugars ranged from 0.42 per cent for 'PBW 534' to 0.62 per cent for ' $\mathrm{WH} 800$ ' in first year. The range observed in second year was from 0.34 per cent for 'PBW 550' and 'PBW 554' to 0.49 per cent for 'PBW 533' and 'PBW 534'. The wheats with good chapati score possessed medium to high values of gluten content which indicated that neither too high nor too low values of gluten, are required to make good chapaties. In first year 'DI 9' showed the lowest (20.10 per cent) and 'PBW 531' showed highest (35.63 per cent) wet gluten contents, where as in second year 'PBW 154' showed lowest (22.80 per cent) and '9D' showed highest (42.07 per cent) of wet gluten content. The dry gluten content of the genotypes was found to vary from 6.27 per cent for 'DI 9" to 11.73 per cent for 'WH 800' in first year. In second year 'PBW 175' showed lowest (7.43 per cent) and '9D' showed highest (14.07 per cent) of dry gluten content. In second year the released varieties with known quality gave lower values than tall wheats. The genetic stocks such as WH 1003, WH 712 and the winter wheat 'Glupro' were having higher gluten index, which corresponds to their high protein content. Commercially released variety PBW 550 and advanced line PBW 554 gave high values of gluten index. The gluten index ranged from 26.44 per cent for ' $C 518$ ' to 87.76 per cent for 'WH 712' in first year, and from 28.10 per cent for 'PBW 175' to 73.13 per cent for 'WH 712' in second year.

Results based on analysis of data from three backcross derived recombinant populations: Significant genotypic differences were observed for chapati score (Table $5 a, b$ and $c$ ) in all the three populations viz: 'A' (C273/PBW343// PBW343), 'B' (C306/PBW534//PBW534) and 'C' (C518/PBW343//PBW343). The data on chapati score and physic-chemical characteristics for the populations is given in table 6,7 and 8 . The range of variation observed for this character in all three populations. Populations ' $A$ ' and ' $C$ ' showed normal distribution of lines for the trait whereas in population ' $\mathrm{B}$ ' it was observed that there was transgression of chapati score on inferior side. In all the three cases, the better parental level ( $C$ series varieties) was not recovered indicating complex control of chapati quality. This absence of superior transgressive segregants was also indicative of fact that positive alleles governing 
chapati quality are largely concentrated in one parent.

In population ' $A$ ' the parental values of chapati score observed were 8.2 and 7.7 for $C 273$ and PBW 343 respectively. None of the genotypes were able to surpass C 273 for chapati score where as 56 out of 70 lines gave a score inferior to PBW 343. For 14 genotypes the score ranged between the parental values. In case of population ' $\mathrm{B}$ ' the parental values observed for chapati score were 8.2 and 7.4 for C 306 and PBW 534 respectively. 22 genotypes out of 70 ranged between the parental values where as remaining 48 gave a score inferior to PBW 534. Similarly in population ' $C$ ' none out of 80 lines were found to outscore C 518 where as 71 genotypes gave a score inferior to PBW 343. Only 9 genotypes gave a chapati score in parental range.

A wide range of variation for grain appearance was observed in the populations. All the three populations were found to be normally distributed with the character being skewed towards higher side. In the recombinant population ' $A$ ' the grain appearance score of the parents was observed to be 5.93 and 5.5 for C 273 and PBW 343 respectively. A total of 32 genotypes out of 70 fell in to this parental range. 30 genotypes were found to be superior to $C 273$ whereas 8 genotypes gave values below PBW 343. In population ' $\mathrm{B}$ ' the parental values observed were 5.77 and 5.53 for $C$ 306 and PBW 534 respectively. 6 genotypes out of 70 gave a score ranging between the parental values, 37 genotypes were found to be superior to C 306 whereas 27 genotypes gave values less than that of PBW 534. In population 'C' only 2 out of 80 genotypes were found to be superior to $C$ 518 (6.17) whereas 59 genotypes gave inferior values than PBW 343 (5.83). 19 genotypes gave values in the parental range. In population ' $A$ ' the parents C 273 and PBW 343 gave a test weight of $76.8 \mathrm{~kg} / \mathrm{hl}$ and $75.0 \mathrm{~kg} / \mathrm{hl}$ respectively. Out of 70 genotypes in this population, 12 were found to have a test weight in the parental range whereas 40 genotypes gave a value above C 273 and 18 genotypes were found to be inferior to PBW 343. In population 'B' only 7 out of 70 genotypes were observed to be superior to C $306(79.67 \mathrm{~kg} / \mathrm{hl})$ and 39 genotypes gave a test weight less than that of PBW 534 (76.67 kg/hl). 24 genotypes gave values in the parental range. In population ' $C$ ' no genotype out of 80 was found to be superior to $C$ $518(81.33 \mathrm{~kg} / \mathrm{hl})$. However 66 genotypes showed a test weight value less than that of PBW 343 (79 $\mathrm{kg} / \mathrm{hl}$ ) and 14 genotypes were found to have a test weight in the parental range.In population ' $A$ ' 41 genotypes out of 70 were observed to have a 1000 grain weight between the parental values of $37.90 \mathrm{~g}$ and $48.56 \mathrm{~g}$ for C 273 and PBW 343 respectively. 5 genotypes gave values above that of PBW 343 whereas 24 genotypes gave a value below that of $C 273$. In population 'B' the 1000 grain weight of the parents C 306 and PBW 534 was observed to be $35.27 \mathrm{~g}$ and $40.75 \mathrm{~g}$ respectively. 28 genotypes out of 70 were having values below that of $C 306$ and 27 genotypes gave value of 1000 grain weight above that of PBW 534. Remaining 15 genotypes were observed to have values between the parental 1000 grain weights. In population ' $C$ ' the parental values of 1000 grain weight were observed to be $33.71 \mathrm{~g}$ and $40.00 \mathrm{~g}$ for C 518 and PBW 343 respectively. 22 genotypes out of 80 were found to have values in between the parental values, whereas 45 genotypes exceeded grain weight than that of PBW 343 and 13 genotypes gave values below that of $C$ 518. None of the genotypes in all the three populations was found to have grain hardness value above the superior value of the donor C-parent. In populations ' $A$ ' and ' $B$ ' 59 out of 70 lines gave a hardness value lower than the recipient parent whereas 11 genotypes gave values in the parental range. The parental values for grain hardness observed were $12.36 \mathrm{~kg} \mathrm{(C} \mathrm{273)} \mathrm{and} 9.51 \mathrm{~kg}$ (PBW 343) for population 'A' and $13.37 \mathrm{~kg}$ (C 306) and $10.59 \mathrm{~kg}$ (PBW 534) for population ' $\mathrm{B}$ '. In 'C' population the 12 genotypes out of 80 ranged between the parental values of $12.31 \mathrm{~kg}$ (C 518) and $10.94 \mathrm{~kg}$ (PBW 343). Remaining 68 lines were found to be inferior to PBW 343. The hard wheat produces granular flour which is desirable for bread making where as soft wheat flour is preferred for biscuit making. Similarly, for chapati making it is prescribed to use medium hard wheat but the situation in India is different. In our wheat the grain hardness is not associated with gluten strength but is simply the physical hardness. Therefore in Indian context the hard wheat varieties produce good chapaties and it has been reported by Mishra (1998) that grain hardness alone contributes about 40 percent to the chapati quality. Upretty and Abrol (1972) pointed out that most of the varieties with acceptable palatability were having high damaged starch content. The authors further noted that damaged starch together with the sugar content should be used as a selection tool for chapati quality. Srivastava et al. (2002) observed that higher moisture retention and starch gelatinization as a consequence of greater film forming ability of gluten in hard wheat flour resulted in pliable and soft textured chapati. Ram et al (2005) emphasized that the good chapati quality wheats like C 306 are likely to differ from present day cultivars in terms of the Pin allele constitution. The phenol reaction score for population ' $A$ ' showed 5 genotypes out of 70 to be better than $C$ 273 (1.87) where as 65 genotypes were found to be inferior to PBW 343 (6.90). None of the genotypes in this population were found to give values in the parental range. However in population ' $B$ ' 63 genotypes out of 70 gave values in the paren- 
tal range of 3.00 (C 306) to 7.77 (PBW 534). 7 genotypes were found to be superior to C 306 whereas no genotype gave phenol score values more than that of PBW 534. Similarly in population ' $C$ ' no genotype out of 80 gave higher phenol score than PBW 343 (5.70) whereas only 5 genotypes were found to be superior to C 518 (1.67). Remaining 75 genotypes were found to have a phenol score in the parental range. In all the three recombinant populations high values of grain protein content were observed for most of the genotypes. In population 'A' 19 genotypes out of 70 gave protein content in the parental range of 12.97 per cent (C 273) and 11.22 per cent (PBW 343). 51 genotypes gave values higher than $C$ 273 whereas as none of the genotypes was found to be inferior to PBW 343. In population 'B' all the 70 genotypes gave a protein content value of more than that of $C 306$ (11.81 per cent). The recipient parent, PBW 534, gave a value of 10.77 per cent grain protein content. In population ' $C$ ' 2 genotypes out of 80 were found to have values ranging between the parental values of 11.95 per cent (C 518) and 10.55 per cent (PBW 343) whereas 78 lines were found to give higher values than C 518.In population 'A' the observed values for sedimentation of parents were observed to be $53.00 \mathrm{cc}$ and $34.67 \mathrm{cc}$ for C 273 and PBW 343 respectively. 10 genotypes out of 70 were found to range between these values whereas 60 genotypes gave values higher than that of $C 273$. In population ' $\mathrm{B}$ ' all the 70 genotypes gave higher values of sedimentation than both the parents $C$ 306 (41.00 cc) and PBW 534 (41.00 cc). None of the genotypes were found to be inferior to any of the parent. In population ' $C$ ' the sedimentation value of 65 genotypes out of 70 was found to be in the parental range of $50.67 \mathrm{Cc}$ (C 518) and 38.33 cc (PBW 343). No genotype inferior to PBW 343 was observed whereas 15 lines gave sedimentation values higher than $C$ 518. The values of diastatic activity were found to be on lower side in all the populations and even none of the lines were found to come close to the parental values. A higher population mean was observed for ' $B$ ' population in comparison to other populations. The lower values of diastatic activity in the populations can be because of lower levels of grain hardness and grain shriveling. The diastatic activity of the recombinant populations ' $A$ ', ' $B$ ' and ' $C$ ' showed that all the genotypes gave higher value than the best parent. The sweetness in chapaties is governed by the diastatic power in addition to the content of sugars in the whole meal. Even Upretty and Abrol (1971) have stressed the need for screening new lines for diastatic power in addition to the sugar content. They also emphasized the role of diastatic power for screening new entries for chapati making.

In population ' $A$ ' the values of falling number ob- served for the parents were 584.00 for C 273 and 600.00 for PBW 343. All the 70 genotypes in the population were found to have falling number values less than that of $C 273$. In population ' $B$ ' the falling number values obtained for $C 306$ and PBW 534 were 499.33 and 578.00 respectively. 49 genotypes out of 70 gave values lower than that of $C 306$ whereas 20 genotypes giving values in the parental range with only 1 line having a value of more than PBW 534. In case of population 'C' 53 genotypes out of 80 gave values in the parental range. 24 genotypes gave lower values of falling number than that of C 518 (480.67) whereas only 3 genotypes gave values above PBW 343 (578.33).In population 'A' the observed values of reducing sugars for the parents were 0.54 per cent for C 273 and 0.55 per cent for PBW 343. 65 out of 70 genotypes gave higher values of reducing sugars than that of PBW 343 whereas only 2 lines were found to be inferior to $C 273$. The values of total sugars observed for C 273 and PBW 343 were 3.40 per cent and 2.92 per cent respectively. In population 'B' 38 genotypes out of 70 gave lower values of reducing sugars than that of C 273 ( 0.51 per cent) and 22 genotypes were found to give lower values than that of PBW 534 ( 0.49 per cent). 10 genotypes ranged between the parental values. The values of total sugars observed for the parents of this population were 3.40 per cent (C 306) and 3.08 per cent (PBW 534). All the 70 genotypes in this population were found to give low total sugars than PBW 534. In population 'C' 50 genotypes out of 80 were found to have lower values of reducing sugars than PBW 343 which had a value of 0.47 per cent. 12 genotypes gave higher values than C 518 ( 0.51 per cent) whereas only 8 genotypes gave values ranging between the parental values. 65 genotypes out of 80 gave values of total sugars ranging between the parental values of 3.40 per cent $(C 518)$ and 3.17 per cent (PBW 343). 8 genotypes gave values lower than that of PBW 343 and 7 genotypes were found to give values higher than C 518 .

In the literature, variable figures have been reported by various workers with respect to the reducing sugars contents of wheat. Singh et al (1983) recorded the content of reducing sugars in whole meal in the range of 0.15 to 0.71 percent based on observations involving improved strains and check varieties.

The reducing sugars content had a significant correlation with the chapati texture after 2 hour storage $(-0.43)$ offering an opportunity to improve keeping quality of chapaties. This implies that that the varieties with a higher content of reducing sugars will produce cookies with a higher spread factor and the chapaties produced by such varieties will remain softer for a longer time. Singh et al (1983) reported non-reducing sugar content in the range of 1.7 to 2.8 percent in respect of the ad- 
vanced genotypes. Singh et al (1983) and Upretty and Abrol (1972) observed that quantity of sugars present in wheat whole meal together with diastatic activity determines the sweetness of chapaties prepared as no sugar is added to the chapaties dough. Hence the sugar content in the whole meal is having a direct relationship with the chapaties prepared.

The gluten content was found to be normally distributed in all the three populations with the genotypes showing a wide range of variation. In population ' $A$ ' the gluten content of the parents was observed to be 10.30 per cent for $C 273$ and 7.90 per cent for PBW 343. 45 out of 70 genotypes in the population were observed to have values of dry gluten content in the parental range. 13 were found to be lower in gluten content than PBW 343 whereas 12 genotypes had values higher than $\mathrm{C}$ 273. The parental values of gluten index in this population were observed to be 41.78 per cent for C 273 and 40.26 per cent for PBW 343. 45 genotypes gave higher gluten index than $C 273$ whereas 18 genotypes were found to have a value lower than PBW 343. Only 7 genotypes ranged between the parental values. In population ' $B$ ' the observed values of dry gluten content of the parents were 9.30 per cent for $C 306$ and 9.80 per cent for PBW 534 . Only 5 genotypes out of 70 gave values in this parental range. 40 were found to be superior to PBW 534 whereas 25 genotypes gave values lower than $C$ 306. The gluten index values in this population showed that 67 genotypes out 70 gave values higher than that of PBW 534 (47.47 per cent) whereas no genotypes gave value lower to C 306 (34.53 per cent). 3 genotypes ranged between the parental values for the gluten index. In population ' $C$ ' the dry gluten index of the parents was 11.37 per cent for C 518 and 7.77 per cent for PBW 343. 64 genotypes out of 80 were found give gluten value in this parental range. 11 were found to give higher values than that of PBW 343 whereas only 5 genotypes gave dry gluten content values of less than that of $C 518$. The gluten index values of the parents in this population were observed to be 19.95 per cent for C 518 and 50.88 per cent for PBW 343. 32 genotypes out of 80 gave gluten index values in this range. 7 genotypes were inferior to C 518 whereas 41 genotypes gave higher values of gluten index than that of PBW 343.

The physico-chemical characteristics are an important group of characters which determine the quality of wheat. The physical character such as grain hardness, test weight, 1000 grain weight, grain appearance score, phenol reaction score, yellow berry incidence etc. and the chemical characteristics such as protein content, gluten content and index, sugar content, diastatic power, sedimentation value, falling number and the amount of pigments in wheat flour are known to affect the wheat end-use quality. Some of the physicochemical characteristics reported to influence the chapati quality are damaged starch content, water absorption of the flour, ash as well as polyphenol oxidase activities (Rao et al 1989, Mallick et al 2013). The similar references regarding the end use quality of wheats have also been achieved in other countries such as Hungary (Diosi et al., 2015), Korea (Kang et al., 2014) and Egypt (ElPorai et al., 2013). The visco-elastic property of dough, which influences the baking quality of wheat, depends on the quality and quantity of protein. Wheat varieties having $9.5-10.5 \%$ protein were found to be suitable for the preparation of chapati (Austin and Ram, 1971). However, it is reported that wheat having higher protein contents $(>12 \%)$ was also found suitable for chapati making, indicating the importance of quality or nature of proteins present in wheat in determining chapati making quality (Srivastava et al., 2003).

Correlation of different traits with chapati score: The first set is not a random collection of genotypes and most of the constituent were chosen with specific considerations as discussed in earlier sections. This may not auger well for association studies but since this set is better characterized in the sense that two years of analysis was carried out. The second set (BC-RIL populations) became available in a stabilized homozygous form towards the end of this study and only one year of analytical work could be conducted. The value of this set lies in the fact that it is an outcome of random segregation of quality traits, thus eliminating incidental correlation and revealing those based on cause and effects. Association of physicochemical characters with chapati traits in set of cultivars and genetic stocks for two years as revealed by genotypic correlation coefficients is given in table 9 and 10.

Grain hardness $(\mathrm{GH})$, which is directly related with damaged starch, showed high positive genotypic correlation with chapati quality in first year. This finding has been reported by a large number of studies (Rao et al., 1989, Srivastava et al., 2003, Hemalatha et al 2007, Inamdar et al 2015, Singh et al., 2016, Panghal et al., 2017). As a result $\mathrm{GH}$ is a widely recognized predictor /selection criteria for chapati quality. A considerable lowering of this correlation was observed in the year second year with genotypic correlation coefficient of 0.55 . The observation of $\mathrm{GH}$ in second year showed a general reduction and narrowing of overall range. Evidently this contributed to lowered correlation. In spite of significant reduction in the $\mathrm{GH}$ in second year the good chapati genotypes such as C 273, C 306, C 591 maintained their superior chapati quality. Further genotypes such as Lok 1 added in the second year displayed superior quality in spite of medium grain hardness. This is also likely to have added to lower correlation in this season. 
Grain appearance based on luster, boldness, shape etc. is often used as a selection criteria and an indicator of consumer preference as well as processing quality. The correlation of grain appearance with chapati quality seems to follow the trend observed for grain hardness, which is in fact a contributing factor to hardness as luster is associated with hardness. The correlation of $0.78 \mathrm{ob}-$ served in first year however differed much more drastically in this case in the second year. Obviously cross over genotypes e.g. those showing good grain appearance but medium chapati score i.e., PBW175 and those showing poor grain quality but above average chapati quality such as Lok 1 , contributed to lacking of this correlation in the second season. In general grain appearance score in second year was low on account of unfavorable environmental conditions leading to poor discrimination of grain appearance characters. A proper expression of grain appearance trait is important for it to serve as an indicator of chapati quality. Also noticeable was the case of 'Glupro', which was not evaluated in second year but in first season it showed very poor grains and at the same time poor chapati score.

Quality character which is often regarded critical for processing is grain protein content. The correlation of protein content with chapati quality however followed an erratic trend in the experiment. The high negative correlation $(-0.78)$ of protein content with chapati quality in first year, was reversed to positive $(0.62)$ in second year. Perusal of the table of means shows several reversals in the ranking of genotypes for protein content between the years. In fact chapati quality showed a greater stability over the years compared to protein content. This is in consonance with the widely recognized pronounced environmental component of protein content. Previous studies are suggestive of the lack of correlations of protein content with chapati quality (Srivastava et al 2003, Hemalatha et al 2007, Hemalatha et al 2016, Harshwardhanet al 2016). Use of protein content as an indicator of chapati quality holds very little potential. First year negative correlation was also influenced by presence of very high protein content genotype 'Glupro' (17.57 per cent) in this set, which showed extremely poor chapati quality.

Another easily observable physical character is yellow berry, which reflects grain internal packages and presence of soft spots. The negative correlation of yellow berry with chapati quality in the first year was probably due to hardly any expression of this trait. Sedimentation value is the most important character for bread making quality and is widely employed as its indicator, needed to be investigated in terms of chapati quality. The correlation value of sedimentation with chapati quality seems to reflect its complete irrelevance for chapati making. This lack of correlation with a key component of bread quality shows that the processing requirements for bread and chapati making may be highly divergent. The correlation trends revealed by other studies (Austin and Ram 1971, Shurpalekar et al 1976, Saxena et al 1997, Srivastava et al 2003,) however showed that the sedimentation values of low to medium range are related to chapati quality. Among the genetic stocks evaluated in the present study, two stocks known for high sedimentation value expressed this trait at high levels in both the seasons, but were consistently medium in terms of their chapati quality.

Not many intrinsic traits are known to relate constantly with chapati quality. Reducing sugars in the study showed high positive and consistent correlation with the chapati quality. Augured over two years reducing sugar content turns out to be the trait with highest correlation with chapati quality. Reducing sugars are directly related to sweetness which contributed 5 out of 35 scoring units, ascribed to overall chapati quality score (converted to 10 in final score). Thus reducing sugars can be used as a suitable objective replacement for the organoleptic assessment of sweetness. Another constituent association has emerged in the form of phenol score. Consistently negative correlation over two years indicates the utility of this easily assessable parameter. The $C$ series varieties, except C 306, had a low phenol score in both seasons. Lowest phenol score of all the genotypes was registered by PBW 175 during second year. Commercial cultivars showed medium to high phenol score.

Gluten (dry and wet) follows a parallel trend with high negative correlation in the first year and high positive correlation in the second year. Both dry and wet gluten follow the same pattern over the years as that of protein content, as was expected. The swing from negative to positive as in case of protein content owes largely to enhanced wet/dry gluten of the C-series varieties in the second year. In the first year the presence of 'Glupro', high gluten and low chapati score wheat, contribute to the negative correlation. As discussed for protein content and sedimentation value, the gluten content also seems to be independent of the chapati quality. Gluten index is an excellent multi trait parameter for predicting bread wheat quality. Greater the proportion of glutenin, in comparison to gliadins, better the bread making quality. The requirement for chapati seems distinctly contrasting. Gluten index had a significant negative correlation with chapati score in first year while weak negative correlation was observed in second year. Most of $\mathrm{C}$-series varieties had a low gluten index. If we see the mean gluten index of cultivars and genetic stocks, none of the lines produced glutenins in excess of gliadins. The high sedimentation line WH 712 reached highest level of 87.76 per cent 
(first year). The good chapati quality wheats ranged between 30-50 per cent gluten index. The negative association of gluten index with chapati quality once again highlights the contrasting requirements for bread and chapati making.

Keeping the red grained 'Glupro' out of the set of genotypes, results in a mild positive correlation of the carotenoids with chapati making quality. This probably is due to moderately high carotenoids content of superior chapati $\mathrm{C}$ series varieties. This level of carotenoids was probably inconsequential to chapati colour and overall chapati score.

The correlations which prevail in the populations carry much greater weight as these have persisted over several rounds of recombination and are likely to reflect under lying causes of superior chapati quality. As various components of chapati quality would be disassembled, the relative levels of correlations for individual traits would be uncovered. The present study in this regard represents an important advance as most of previous correlation studies were based on set of cultivars.

Valuable correlation trends which extend across the three populations (Table 11) may be summarized as follows:

Grain hardness seems to have a clear role in chapati quality with a correlation coefficient of 0.34 and 0.35 observed in populations $A$ and $B$ and $a$ value of correlation coefficient in population $C$ is lower at 0.17 .

More consistent correlation was found for grain appearance ranging from 0.26 for $A$ to 0.36 for population B.

Protein content and sedimentation value are clearly out of reckoning as chapati making traits on account of non significant correlation values.

Consistent high positive correlations have showed up for diastase activity, which ranged from 0.32 for $A$ to 0.41 for population $C$ and 0.46 for population $\mathrm{B}$. this consistent behaviour is a strong evidence for the role of this trait in chapati making quality.

The sugars (reducing and total) though less consistent have about half of the 6 correlation values in the zone of significance. A greater role has been ascribed to this trait in the set of cultivars.

The phenol score is a trait which fails the test of strong correlation when observed in the recombinant populations. Much higher and consistent correlation had been observed in the set of cultivars and stocks. The recombinant populations reveal the likely pitfall of using this trait as an index of chapati quality.

The wet and dry gluten fall below significance threshold in the populations $B$ and $C$. in population A where a positive correlation is observed, it is likely to be due to the high gluten index of superior chapati parent ' $C$ 273'. The gluten index remains in consistent, though mild negative correlation with chapati quality.

\section{Conclusion}

The results obtained in the present study have been repetitive as these have been derived from three parallel experiments on three recombinant populations. Past studies on chapati making quality have focussed on genotypic evaluation and hence there was bound to be a bias in the correlation landscape generated by the set of cultivars and genetic stocks. The recombinant populations have helped in threshing out results and hence the correlations look more realistic. Correlations of hardness and grain appearance to chapati quality are toned down to more realistic values. Diastase activity emerges as a more consistent and stronger contributor to chapati making quality. Phenol score may not serve as a suitable indicator of chapati quality. This study has added significantly to the understanding of the basis of chapati making quality in wheat. The results derived based on two sets of plant material have authenticated the correlations between different quality parameters and the end use quality. Validation of present results needs to be carried out for different genotypes, as the chapati quality being a complex trait is highly influenced by environment, agricultural inputs etc.

\section{REFERENCES}

AACC (1990). Approved Methods of American Association of Cereal Chemists, St. Paul, Minnesota, USA

Ahmed, R., Ali, R., Khan, M.S., Sayeed, S.A., Saeed, J. and Yousufi, F. (2015). Effect of proteases and carbohydarse on dough Rheology and End quality of cookie. American J Food Sci. Nutr. Res., 2(2): 62-66.

Axford, D.W.E., McDermott, E.E. and Radman, D.G. (1979). Note on sodium dodecyle sulphate test of bread making quality comparison with Pelshenke and Zeleny tests. Cereal Chem.,56: 582-584.

Austin, A. and Ram, A. (1971). Studies on chapati making quality of wheat. Rajendra Printers, Ram Nagar, New Delhi, pp 1-108.

Diosi, G., More, M. and Sipos, P. (2015). Role of Farinograph test in the wheat flour quality determination. Acta Universitatis Sapientiae Alimentaria 8: 104-110.

El-Porai, E.S., Salama, A.E., Sharaf, A.M., Hegazy, A.I. and Gadallah, M.G.E. (2013). Effect of different milling processes on Egyptian wheat flour properties and pan bread quality. Ann. Agricul. Sci. 58(1): 51-59.

Finney, P.L., Bains, G.S., Hoseney, R.C. and Lineback, D.R. (1973). Quality of Indian wheats. Cereal Sci. Today, 18(12): 392-397.

Gupta, P.K., Varshney, R.K., Sharma, P.C. and Ramesh, B. (1999). Molecular makers and their applications in wheat breeding. Pl. Breed.,118: 369-390.

Gill, B.S., Singh, N. and Sodhi, N.S. (2006). Studies on physico-chemical, textural and functional properties of wheat flour from different Indian cultivars. J. Food Sci. Technol., 43: 56-64.

Harshwardhan, Kumar, A., Kumar, A. and Prasad, B. (2016). Study of variance, heritability and genetic advance for various yield contributing and quality traits in spring wheat (Triticum aestivum L.). J. Appl. Nat. Sci., 8(4): 1811-1814.

Hemalatha, M.S., Manu, B.T., Bhagwat, S.G., Leelava- 
thi, K. and Rao, U.J.S.P. (2007). Protein characteristics and peroxidase activities of different Indian wheat varieties and their relationship to chapatimaking quality. European Food Res. Technol.,225: 463-471.

Hemalatha, M., Manohar, R.S., Salimath, P.S. and Rao, U.J.S.P. (2013). Effect of Added Arabinoxylans Isolated from Good and Poor Chapati Making Wheat Varieties on Rheological Properties of Dough and Chapati Making Quality. Food Nutri. Sci., 4: 884-892.

Inamdar, A.A., Sakhare, S.D. and Prabhasankar, P. (2015). Chapati Making Quality of Whole Wheat Flour (Atta) Obtained by Various Processing Techniques. J. Food Process. Preserv., 39: (6) 30323039.

Irvine, G.N. and Bains, G.S. (1965). Studies on the qualities of some improved varieties of Indian wheats J. Sci. Food Agri., 16: 256-257.

Kang, C.S., Jung, J.U., Baik, B.K. and Park, C.S. (2014). Relationship between physicochemical characteristics of flour and sugar snap cookie quality in Korean wheat cultivar. Internatl. Food Res. J., 21(2): 617-624.

Kumar, S., Sohu, V.S. and Bains, N.S. (2018). Agronomic performance of Indian wheat varieties and genetic stocks known for outstanding chapati quality characteristics. J. Appl. Natrl. Sci., 9(1): in press.

Mallick, S.A., Azaz, K., Gupta, M., Sharma, V. and Sinha, B.K. (2013). Characterization of grain nutritional quality in wheat. Indian J. PI. Physiol., 18(2):183-186

Mishra, B.K. (1998). Quality needs for Indian traditional products. In: Wheat: Research Needs Beyond 2000 $A D$. Narosa Publishing House, New Delhi.

Naik, H.R., Sekhon, K.S. and Abbas Wani, A. (2010). Physicochemical and dough handling characteristics of Indian wheat and triticale cultivars. Food Sci. Technol. International, 16(5): 371-379.

Panghal, A., Chhikara, N. and Khatkar, B.S. (2017). Characterization of Indian wheat varieties for chapati (flat bread) quality. J. Saudi Society Agril. Sci., http:// dx.doi.org/10.1016/j.jssas.2017.02.005.

Rao-Haridas, P., Leelvathi, K. and Shurpalekar, S.R. (1989). Effect of damaged starch on the chapati making quality of whole wheat flour. Cereal Chem.,66: 329-333.

Ram, S., Jain, N., Sharon, J. and Singh, R. (2005). New frame shift mutation in puroindoline $B$ in Indian wheat cultivars Hyb65 and NI5439. J. Pl. Biochem. Biotech., 14:45-48.

Saxena, D.C., Rao, U.J.S.P. and Rao, P.H. (1997). Indian wheat cultivars: correlation between quality of gluten proteins, rheological characteristics of dough and tandoori roti quality. J. Sci. Food Agric.,74:265272.

Sekhon, K.S., Bakshi, A.K., Sehgal, K.L. and Gill, K.S. (1976). Studies on the physico chemical and rheological characteristics of some improved wheat verities. Indian J. Biochem. Biopys., 13: 22.

Shurpalekar, S.R., Kumar, G.V., Venkateswara, R.G., Ranga Rao, G.C.P., Rahim, A. and Vatsala, C.N. (1976). Physico-chemical, rheological and mixing characteristics of bread and chapati making quality of Indian wheats. J. Food Sci. Tech.,13: 79-86.

Sidhu, J.S., Seibel, W., Brummer, J.M. and Zwingelberg, $H$. (1988). Effect of flour milling conditions on the quality of Indian unleavened flat bread (Chapati). J. Food Sci.,53: 1563-1565.

Singh, R. and Bailey, C.H. (1940). A biochemical and technological study of Punjab wheat varieties Cereal Chem., 17:169.

Singh, R.P., Singh, R.P. and Bains, G.S. (1978). A study of interaction of bread improvers on rheological and baking properties of Punjab wheats. J. Food Sci. Tech.,15: 255-258.

Singh, S., Verma, A. and Bala, N. (2016). Sensory and nutritional evaluation of unleavened flat bread prepared by multigrain flour mixture. J. Appl. Natrl. Sci., 8(3): 1168-1171.

Singh, R.P., Sehgal, K.L., Saxena, A.K., Sekhon, K.S and Gill, K.S. (1983). Physico chemical, rheological and baking characteristics of some promising strains of wheat. J. Res. Punjab Agric. Univ., 20: 403-410.

Srivastava, A.K., Meyer, D., Rao, P.H. and Seibel, W. (2002). Scanning electron microscopic study of dough and chapati from gluten-reconstituted good and poor quality flour. J. Cereal Sci.,35: 119-128.

Srivastava, A.K., Rao, J.S.P. and Rao, P.H. (2003). Studies on protein and its high molecular weight subunit composition in relation to chapati making quality of Indian wheat cultivars. J. Sci. Food Agric., 83: 225-231.

Supekar, D.T., Patil, S.R. and Munjal, S.V. (2005). Comaprative study of some important aestivum, durum and dicoccum wheat cultivars for grain, flour quality and suitability for chapati making characteristics. $J$. Food Sci. Technol., 42: 488-492.

Syed, H.M. (1990). Indian wheats in relation to Chapati: a review. Indian Miller, 21: 17-20.

Syed H M, Rathi S D, Swarate A R and Taur A T (1991) Studies on bread making potential. Indian Miller 22: 25-29.

Upretty, D.C. and Abrol, Y.P. (1972). Studies on Chapati quality. Bull. Grain Tech., 10: 276-282. 